\title{
Mechanics of Static Slip and Energy Dissipation in Sandwich Structures: Case of Homogeneous Elastic Beams in Transverse Magnetic Fields
}

\author{
Charles A. Osheku \\ Centre for Space Transport and Propulsion, National Space Research and Development Agency, \\ Federal Ministry of Science and Technology, FCT, Abuja, PMB 437, Nigeria \\ Correspondence should be addressed to Charles A. Osheku, charlesosheku2002@yahoo.com
}

Received 26 June 2012; Accepted 13 August 2012

Academic Editors: K. Abhary, J. Clayton, and K. Ismail

Copyright () 2012 Charles A. Osheku. This is an open access article distributed under the Creative Commons Attribution License, which permits unrestricted use, distribution, and reproduction in any medium, provided the original work is properly cited.

\begin{abstract}
Mechanics of static slip and energy dissipation in sandwich structures with respect to two-layer homogeneous elastic beams in a transverse magnetic field is presented. The mathematical physics problem derives from nonuniform contact conditions of press sandwich layers or joints. On this theory, equations governing the stresses and the deflection profile are derived. By restricting analysis to the case of cantilever architecture, closed form polynomial expressions are computed for the deflection, interfacial slip, slip, and strain energies of the system. In particular, the effects of magnetoelasticity and interfacial pressure gradient on these properties are demonstrated for design analysis and engineering applications. In addition, explicit mathematical equations couched in magnetoelasticity and pressure gradient polynomial kernels with fractional coefficients for critical values of pressure for which no slip occurs at the tip and the optimum clamping pressure for optimal slip energy dissipation are derived. It is also shown for special cases that recent results in literature are recoverable from the theory reported in this paper.
\end{abstract}

\section{Introduction}

Investigation into the vibration and magnetoelastic stability of ferromagnetic flexible structures, beams, beam-plates, plates, and shells is abound in literature. Concerning these theoretical analyses or experimental studies, comprehensive reviews of trends are reported in [1-18]. For theoretical analyses, the effect of eddy current in the ferromagnetic material was neglected. Lee [1] in contrast to earlier investigators studied the dynamic stability with magnetic damping arising from eddy current and derived an explicit expression for the destabilizing effect. For experimental investigation, Moon and Pao [2] were credited with the pioneering work on magnetoelastic buckling of a ferromagnetic thin plate in transverse magnetic fields. Their results showed that a ferromagnetic plate buckles and loses its stability when the magnetic intensity approaches a critical value that is functionally related to the geometric ratio of length to plate thickness via a $3 / 2$ power law. Based on these findings, a mathematical problem was contrived as the magnetic body coupled model to predict the experimental phenomenon of magnetoelastic instability and critical magnetic field. Additional experiment later showed that the natural frequency of a beam-plate decreased with increasing magnetic field intensity and becomes near to zero, as the field attains a critical value, which causes the same beam-plate to buckle statically. Following the emergence of large discrepancy between the theoretical predictions and experimental results of Moon and Pao in [3], research attentions were further devoted to the study of magnetoelastic stability and buckling problems.

Some investigators, Wallerstein and Peach [4], Miya et al. [5], Peach et al. [6], and so forth, directed their attentions to finding satisfactory explanations for these discrepancies. Notwithstanding the significance of previous findings, Lee [7] investigated the dynamic stability of electrically conducting beam-plates in transverse magnetic fields via a concise theory of flexural vibration of magnetoelastic plates immersed in transverse magnetic fields. Similarly, in the 1990s, additional theories were developed for the study of magnetoelastic buckling and bending of ferromagnetic plates 
in transverse and/or oblique magnetic fields via a generalized variational principle of magnetoelasticity by Zhou et al. [8], Zhou and Zheng [9], and Zhou and Miya [10]. In 2002, the experimental results of Wang et al. [11] confirmed the theoretical predictions in the 1990s.

Following renewed interest in magnetoelasticity and its applications in engineering systems, namely, magnetic storage elements, magnetic structural devices, geophysical physics, and plasma physics, attentions were directed on the study of magnetothermodynamic stress and perturbation of magnetic field vector in both solid and orthotropic thermoelastic cylinders. In this regard, Wang et al. [12] employed finite integral transforms to examine theoretically the magnetothermoelastic waves and perturbation of the magnetic field vector produced by thermal shock in a solid conducting cylinder. In this study, closed forms expressions were derived for magnetothermodynamic stress and perturbation response of an axial magnetic field vector in a solid cylinder. Comprehensively, Wang et al. [13] investigated the magnetothermoelastic responses and perturbation of the magnetic field vector in a conducting orthotropic thermoelastic cylinder subjected to thermal shock using finite Hankel integral transform, whilst Librescu et al. [14] and Wang et al. [15] studied the effect of magnetothermoelasticity of ferromagnetic conducting plates under excitations theoretically.

In related development, Wang and Dai [16] investigated the dynamic responses of piezoelectric hollow cylinders in axial magnetic field. This study led to the development of a concise analytical solution to reveal the interaction between mechanical and electromagnetoelastics responses of piezoelectric hollow cylinders subjected to arbitrary mechanical loadings and electric potential shock. An interpolation method was employed to solve the resulting Volterra integral equation of the second kind, arising from interaction between different physical fields. Furthermore, closed forms results were derived for dynamic stresses, electricdisplacements and electric-potentials as well as perturbation responses using finite and Laplace integral transforms.

Meanwhile, the problem of stability loss and free vibration of electromagnetically conducting plate conveying an electric current in magnetic field environment was investigated by Hansanyan et al. [17]. Following the theoretical models in the 1990s, Wang and Lee [18] considered the magnetic damping effect induced by the eddy current and its effect on dynamic stability. Application of these structures is receiving significant attentions in magnetic propulsion devices for space transport and exploration. From experimental investigations, ferromagnetic flexible structures are usually subjected to magnetic forces arising from the coupling or mutual influence of the magnetization and magnetic fields.

Following recent advances in the mechanics of sandwich layered elastic structures, in an environment of nonuniform interface pressure by Damisa et al. [19, 20], Olunloyo et al. [21], Olunloyo et al. [22], and Osheku and Damisa [23] investigated the flexural vibration of a two-layer magnetoelastic beam in a transverse magnetic field. In their study, equations of mathematical physics governing the stresses and the structural vibration were derived via laminated beam theory employing Newtonian form of Cauchy's stress equations.

Although the study was restricted to the case of cantilever structure, the effects of magnetoelasticity, material conductivity, and interfacial pressure gradient on the system response were computed in the form of polynomial expression via Laplace and finite Fourier integral transforms.

The study also shows that each mode of vibration was governed by a two-dimensional family of natural frequencies. The natures of the closed forms expressions for the natural frequencies indicate that the oscillation ceases when the two become simultaneously zero. In both theory and experiment, this is the required condition for static or quasistatic buckling of any layered elastic structure in a transverse magnetic field.

In fact, it is an indication that with suitable geometric parameters and matching transverse magnetic field, critical damping can be enhanced. For special and limit cases, recent theoretical and experimental results were validated. Following the increasing significant of studying both theoretically and experimentally the required condition for static or quasistatic buckling of any layered elastic structure in a transverse magnetic field, this study is devoted to the comprehensive investigation of the characteristics of statically loaded homogenous two-layer sandwich magnetoelastic cantilever structure in a transverse magnetic field.

This paper is organized as follows. Section 1 introduces the problem under investigation within a general context. In Section 2, the essential analytical mechanics leading to the mathematical physics problem with additional specialized static boundary values ordinary differential equations are presented. In Section 3, formal analysis of the problem of interest using finite Fourier integral transform is discussed. Section 4 is concerned with the analysis of static slip, whilst in Section 5 the energy dissipation ability and damping capacity of the structure are analysed. In Section 6, simulated results are discussed. Finally, the paper ends with conclusion in Section 7.

\section{Formulation of the Governing Differential Equation Problem Definition}

As illustrated in Figure 1(a), the problem here is to examine analytically the effect of the pressure gradient on the damping properties of a statically loaded two-layer magnetoelastic beams clamped together in an environment of nonuniform pressure.

2.1. Underlying Assumptions. A two-layer elastic structure is subject to a transverse magnetic field. For the contrived structure, the upper and the lower layers are assumed to be perfectly press fit surfaces of homogenous magnetoelastic beams. The contact conditions between the mating layers as itemized in Damisa et al. [20] hold, namely;

(i) there is continuity of stress distributions at the interface to sufficiently hold the separate layers together both in the pre- and postslip conditions;

(ii) the static deflection of each beam is small compared with the span; 


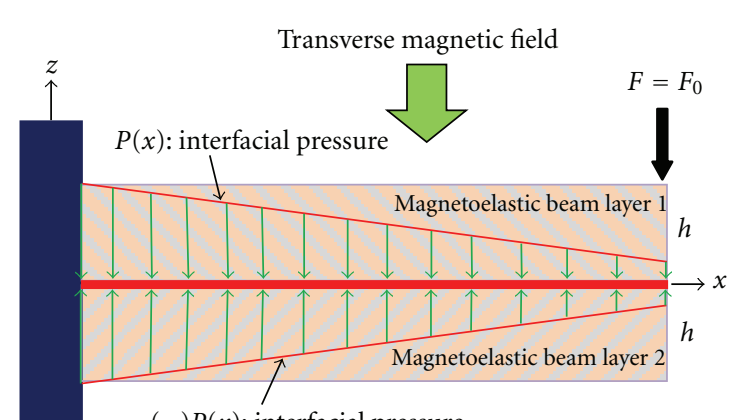

$(-) P(x)$ : interfacial pressure

(a)

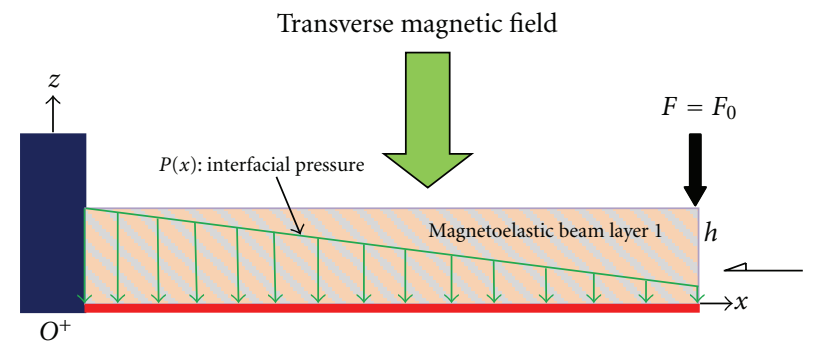

(c)

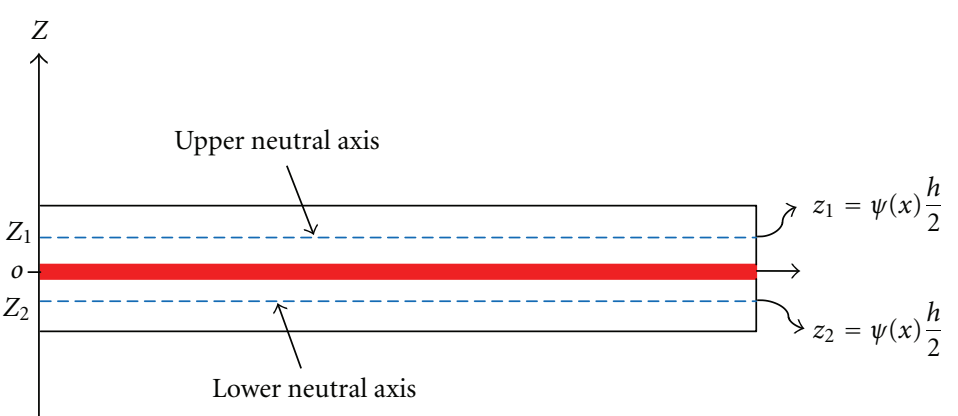

(b)

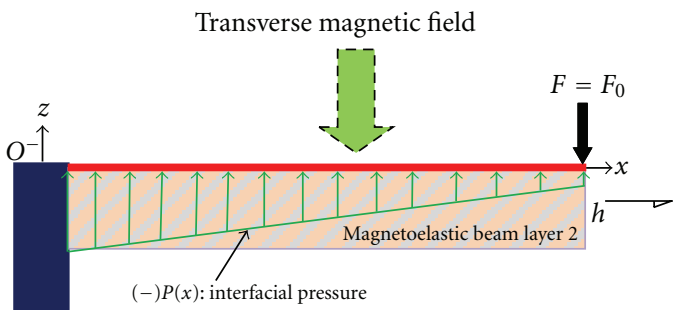

(d)

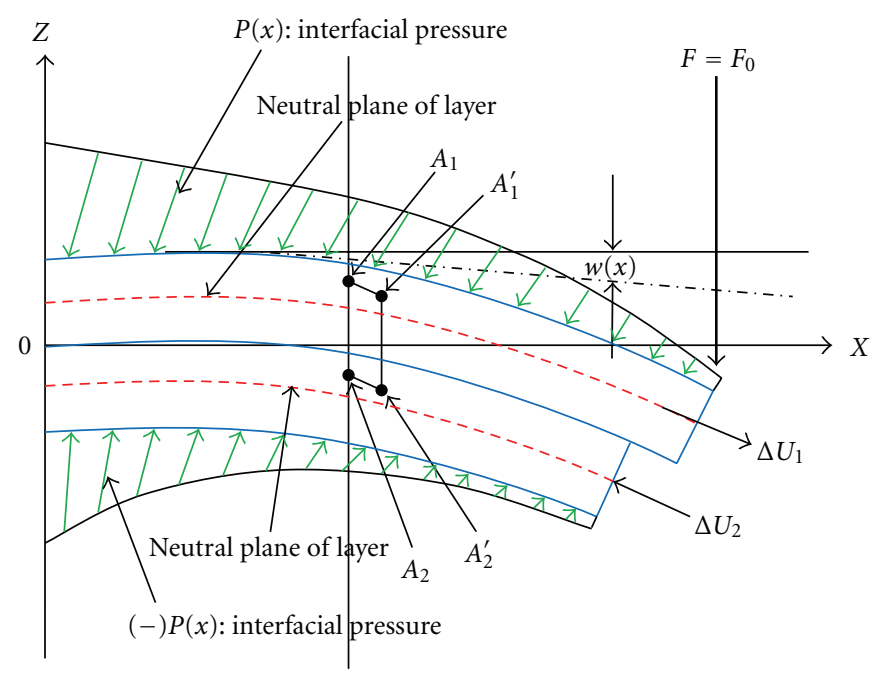

(e)

FIGURE 1: (a) Preslip geometry for the sandwich structure under static load. (b) conceptual description of the upper and lower layers neutral axes. (c) upper layer postslip geometry under static load. (d) lower layer postslip geometry under static load. (e) mechanism of interfacial slip geometry.

(iii) during bending, the magnetoelastic structure has (upper and lower) layers such that each has its neutral plane which may not necessarily coincide with its geometric mid plane of the resultant structure. These neutral planes are located at $z_{1}=\psi(x)(h / 2)$ and $z_{2}=-\psi(x)(h / 2)$, where $\psi(x)$ is a function of $x$ as illustrated in Figure 1(b);

(iv) the approximations involved in the forgoing beam theory are such that the field variables are linear and are expressible in terms of the derivatives of the transverse static deflection $W(x)$ and is taken to be same for both layers.

By defining $u(x, z)$ and $W(x)$ as displacements along $x$ and $z$, respectively, the following relations hold from the classical theory of elasticity, namely,

$$
\varepsilon_{x}=\frac{d u}{d x} ; \quad \gamma_{x z}=\left(\frac{d W}{d x}+\frac{d u}{d z}\right)=0,
$$

$\frac{d u}{d z}=-\frac{d W}{d x}$ such that: $\int_{U_{01}}^{U_{1}} \frac{d u}{d z} d z=\int_{U_{01}}^{U_{1}} d u=-\int_{Z_{1}}^{Z_{0}} \frac{d W}{d x} d z$. 
Equation (2) can be evaluated as

$$
U_{1}-U_{01}=-\int_{Z_{0}}^{Z_{1}} \frac{d W}{d x} d z=-\left(z_{0}-z_{1}\right) \frac{d W}{d x}
$$

Now, $\forall z_{0}=z ; z_{1}=\psi(x)(h / 2)$, following assumption (iii) above, we can rewrite (3) as

$$
\begin{aligned}
U_{1}-U_{01}=-\int_{Z_{0}}^{Z_{1}} \frac{d W}{d x} d z & =-\left(z_{0}-z_{1}\right) \frac{d W}{d x} \\
& =-\left(z-\psi(x) \frac{h}{2}\right) \frac{d W}{d x}
\end{aligned}
$$

to obtain the following expression:

$$
U_{1}=-\left(z-\psi(x) \frac{h}{2}\right) \frac{d W}{d x}+U_{01}(x)
$$

where $U_{01}(x)$ is the point of initiation of interfacial slip in the upper layer.

Similarly, the following expression holds for the lower layer as

$$
U_{2}-U_{02}=-\int_{Z_{0}}^{Z_{12}} \frac{d W}{d x} d z=-\left(z_{0}-z_{2}\right) \frac{d W}{d x} \quad \forall z=-\psi(x) \frac{h}{2}
$$

and evaluated to obtain the following expression:

$$
U_{2}=-\left(z+\psi(x) \frac{h}{2}\right) \frac{d W}{d x}+U_{02}(x),
$$

where $U_{02}(x)$ admits same definition in the lower layer.

From classical theory of elasticity, the in-plane bending stress for the upper layer takes the following form:

$$
\sigma_{x}(x, z)=\sigma_{x}(x, z)_{1}=E \varepsilon_{x 1}=E \frac{d U_{1}}{d x} .
$$

On substituting (5), the foregoing becomes

$$
\begin{aligned}
& \sigma_{x}(x, z)_{1} \\
& =E \frac{d U_{1}}{d x} \\
& =-E\left(\frac{d}{d x}\left(\left(z-\psi(x) \frac{h}{2}\right) \frac{d W}{d x}+U_{01}(x)\right)\right)
\end{aligned}
$$

$$
\begin{aligned}
& =-E\left(\frac{d}{d x}\left(z-\psi(x) \frac{h}{2}\right) \frac{d W}{d x}+\left(z-\psi(x) \frac{h}{2}\right) \frac{d^{2} W}{d x^{2}}+\frac{d U_{01}(x)}{d x}\right) \\
& =-E\left(\left(-\frac{h}{2}\left(\frac{d \psi(x)}{d x}\right) \frac{d W}{d x}\right)+\left(z-\psi(x) \frac{h}{2}\right) \frac{d^{2} W}{d x^{2}}+\frac{d U_{01}(x)}{d x}\right) .
\end{aligned}
$$

Now $-(h / 2)(d \psi(x) / d x)(d W / d x)$ is a nonlinear term, whilst $d U_{01}(x) / d x$ is the strain at the point of initiation of static slip.

Following assumption (iv) (linear theory), $-(h / 2)$ $(d \psi(x) / d x)(d W / d x)$ is negligible while $d U_{01}(x) / d x=0$ at the fixed end. Consequently,

$$
\begin{aligned}
& \sigma_{x}(x, z)_{1} \\
& =E \frac{d U_{1}}{d x} \\
& =-E\left(\frac{d}{d x}\left(\left(z-\psi(x) \frac{h}{2}\right) \frac{d W}{d x}+U_{01}(x)\right)\right) \\
& =-E\left(\frac{d}{d x}\left(z-\psi(x) \frac{h}{2}\right) \frac{d W}{d x}\right. \\
& \left.\quad+\left(z-\psi(x) \frac{h}{2}\right) \frac{d^{2} W}{d x^{2}}+\frac{d U_{01}(x)}{d x}\right) \\
& =-E\left(z-\psi(x) \frac{h}{2}\right) \frac{d^{2} W}{d x^{2}} .
\end{aligned}
$$

Similarly for layer (2), we have

$$
\sigma_{x}(x, z)=\sigma_{x}(x, z)_{2}=E \varepsilon_{x 2}=E \frac{d U_{2}}{d x} .
$$

On substituting $U_{2}=-(z+\psi(x)(h / 2))(\partial W / \partial x)+U_{02}(x)$, (11) becomes

$$
\begin{aligned}
& \sigma_{x}(x, z)_{2} \\
& =E \frac{d U_{2}}{d x} \\
& =-E\left(\frac{d}{d x}\left(\left(z+\psi(x) \frac{h}{2}\right) \frac{d W}{d x}+U_{02}(x)\right)\right) \\
& =-E\left(\frac{d}{d x}\left(z+\psi(x) \frac{h}{2}\right) \frac{d W}{d x}+\left(z+\psi(x) \frac{h}{2}\right) \frac{d^{2} W}{d x^{2}}+\frac{d U_{02}(x)}{d x}\right) \\
& =-E\left(\left(\frac{h}{2}\left(\frac{d \psi(x)}{d x}\right) \frac{d W}{d x}\right)+\left(z+\psi(x) \frac{h}{2}\right) \frac{d^{2} W}{d x^{2}}+\frac{d U_{02}(x)}{d x}\right) .
\end{aligned}
$$


Following assumption (iv) (linear theory), $(h / 2)(d \psi(x) / d x) \times$ $(d W / d x)$ is negligible while $d U_{02}(x) / d x=0$ at the fixed end. Consequently,

$$
\begin{aligned}
& \sigma_{x}(x, z)_{2} \\
& =E \frac{d U_{2}}{d x} \\
& =-E\left(\frac{d}{d x}\left(\left(z+\psi(x) \frac{h}{2}\right) \frac{d W}{d x}+U_{02}(x)\right)\right) \\
& =-E\left(\frac{d}{d x}\left(z+\psi(x) \frac{h}{2}\right) \frac{d W}{d x}\right. \\
& \left.\quad+\left(z+\psi(x) \frac{h}{2}\right) \frac{d^{2} W}{d x^{2}}+\frac{d U_{02}(x)}{d x}\right) \\
& =-E\left(z+\psi(x) \frac{h}{2}\right) \frac{d^{2} W}{d x^{2}} .
\end{aligned}
$$

Next, we invoke the static form of the generalized Cauchy stress equation in the absence of body forces, namely,

$$
\nabla \cdot \vec{\tau}=0
$$

where $\vec{\tau}$ is the stress tensor.

In the upper and lower halves, (14) admits the following forms:

$$
\begin{gathered}
\frac{\partial}{\partial x} \sigma_{(x) 1}+\frac{\partial}{\partial z} \tau_{(x z) 1}=0, \\
\frac{\partial}{\partial z} \sigma_{(z) 1}+\frac{\partial}{\partial x} \tau_{(x z) 1}=0, \\
\frac{\partial}{\partial x} \sigma_{(x) 2}+\frac{\partial}{\partial z} \tau_{(x z) 2}=0, \\
\frac{\partial}{\partial z} \sigma_{(z) 2}+\frac{\partial}{\partial x} \tau_{(x z) 2}=0 .
\end{gathered}
$$

On substitution of (10), (13), we rewrite the above as

$$
\begin{aligned}
& -E z \frac{d^{3} W}{d x^{3}}+\frac{E h}{2} \frac{d}{d x}\left(\psi(x)\left(\frac{d^{2} W}{d x^{2}}\right)\right)+\frac{d \tau_{(x z) 1}}{d z}=0, \\
& -E z \frac{d^{3} W}{d x^{3}}-\frac{E h}{2} \frac{d}{d x}\left(\psi(x)\left(\frac{d^{2} W}{d x^{2}}\right)\right)+\frac{d \tau_{(x z) 2}}{d z}=0 .
\end{aligned}
$$

Following Goodman and Klumpp [24], (16)-(17) must satisfy the following postslip boundary conditions along $x z$ plane, namely,

$$
\begin{gathered}
\sigma_{(z) 1}(x, 0)=-p(x, 0) ; \quad \sigma_{(z) 2}(x,-h)=p(x, 0) ; \\
\tau_{(x z) 1}(x, h)=0 ; \quad \tau_{(x z) 2}(x,-h)=0 ; \\
\tau_{(x z) 1}^{2}(x, 0)=\mu^{2} \sigma_{(z) 1}^{2}(x, 0) ; \quad \tau_{(x z) 2}^{2}(x, 0)=\mu^{2} \sigma_{(z) 2}^{2}(x, 0) ; \\
\int_{0}^{h} \sigma_{(x) 1}(x, z) d z=\int_{0}^{h} z \sigma_{(x) 1}(x, z) d z=0 ; \\
\int_{-h}^{0} \sigma_{(x) 2}(x, z) d z=\int_{-h}^{0} \sigma_{(x) 2}(x, z) d z=0 .
\end{gathered}
$$

From Lee [7], the in-plane shear stress arising from electromagnetic surface traction follows from the generalized Maxwell's stress tensor $\tau^{M}$ defined as

$$
\begin{aligned}
\tau^{M}= & \mu_{M} \vec{H} \otimes \vec{H}+\varepsilon_{m} \vec{E}_{m} \otimes \vec{E}_{m} \\
& -\frac{1}{2}\left(\mu_{m} \vec{H} \cdot \vec{H}+\varepsilon_{m} \vec{E}_{m} \cdot \vec{E}_{m}\right) I_{s} .
\end{aligned}
$$

By enforcing small perturbation on the primary bias field due to the field- structure interaction, following Lee [7], the following expression ensues

$$
\vec{H}(x)=\vec{H}_{0}+\vec{h}_{1}(x) ; \quad \vec{E}_{M}(x)=0+\vec{e}(x) .
$$

Here the field quantities in lower-case letters are assumed to be small magnetic and electric perturbation variables. Consequently, their products can be neglected. Under this circumstance, the Maxwell's stress tensor in (19) reduces to the form

$$
\tau^{M}=\left(\vec{B}_{0} \otimes \vec{h}_{1}+\vec{b}_{m} \otimes \vec{H}_{0}\right)-\frac{1}{2}\left(\vec{B}_{0} \cdot \vec{h}_{1}+\vec{b}_{m} \cdot \vec{H}_{0}\right) I_{s}
$$

Utilizing the relations in Lee [7], the in-plane and out-plane static frictional stresses are modified as

$$
\begin{gathered}
\tau_{(x z) 1}(x, 0)=\mu p(x, 0)-\frac{2}{\mu_{0}}\left(1-\frac{\mu_{0}}{\mu_{m}}\right) B_{0}{ }^{2} \frac{d W}{d x}, \\
\tau_{(x z) 2}(x, 0)=-\mu p(x, 0)-\frac{2}{\mu_{0}}\left(1-\frac{\mu_{0}}{\mu_{m}}\right) B_{0}{ }^{2} \frac{d W}{d x} .
\end{gathered}
$$

Consequently, (16) can be integrated to obtain

$$
\begin{aligned}
& \tau_{(x z) 1}(x, h) \\
& =\left(\begin{array}{l}
\frac{E\left(z^{2}-z h\right)}{2} \frac{d^{3} W}{d x^{3}}+\frac{2}{\mu_{0}}\left(1-\frac{\mu_{0}}{\mu_{m}}\right) B_{0}{ }^{2} \frac{(z-h)}{h} \frac{d W}{d x} \\
-\frac{\mu p(x, 0)(z-h)}{h}
\end{array}\right) .
\end{aligned}
$$

Similar expression can be derived for the lower layer as

$$
\begin{aligned}
\tau_{(x z) 2}(x, h) \\
=\left(\begin{array}{l}
\frac{E\left(z^{2}+z h\right)}{2} \frac{d^{3} W}{d x^{3}}+\frac{2}{\mu_{0}}\left(1-\frac{\mu_{0}}{\mu_{m}}\right) B_{0}{ }^{2} \frac{(z+h)}{h} \frac{d W}{d x} \\
+\frac{\mu p(x, 0)(z+h)}{h}
\end{array}\right) .
\end{aligned}
$$

On substituting (23)-(24) into the second parts of (15a)(15b) the generalized ordinary differential equation governing the static deflection of the sandwich magnetoelastic structure is.

$$
E I \frac{d^{4} W}{d x^{4}}+\frac{b h}{\mu_{0}}\left(1-\frac{\mu_{0}}{\mu_{m}}\right) B_{0}{ }^{2} \frac{d^{2} W}{d x^{2}}=\frac{1}{2} b h \mu \frac{d P}{d x} .
$$

The following specialized ordinary differential equations can be formulated from the foregoing as follows. 
Case 1. For the case of a beam-plate of thickness $h,(25)$ becomes

$$
\begin{gathered}
E I^{*} \frac{d^{4} W}{d x^{4}}+\frac{b h}{\mu_{0}}\left(1-\frac{\mu_{0}}{\mu_{m}}\right) B_{0}{ }^{2} \frac{d^{2} W}{d x^{2}} \\
=\frac{1}{2} b h \mu \frac{d P}{d x} \quad \forall I^{*}=\frac{b h^{3}}{12\left(1-v^{2}\right)} .
\end{gathered}
$$

By dropping the variable $b$, the formulated equation governing the static deflection takes the form

$$
\begin{aligned}
D \frac{d^{4} W}{d x^{4}} & +\frac{h}{\mu_{0}}\left(1-\frac{\mu_{0}}{\mu_{m}}\right) B_{0}{ }^{2} \frac{d^{2} W}{d x^{2}} \\
= & \frac{1}{2} h \mu \frac{d P}{d x} \quad \forall D=\frac{E h^{3}}{12\left(1-v^{2}\right)} .
\end{aligned}
$$

Case 2. For the case of a beam-plate of thickness $2 h,(25)$ becomes

$$
\begin{aligned}
& E I^{* *} \frac{d^{4} W}{d x^{4}}+\frac{b h}{\mu_{0}}\left(1-\frac{\mu_{0}}{\mu_{m}}\right) B_{0}{ }^{2} \frac{d^{2} W}{d x^{2}} \\
& \quad=\frac{1}{2} b h \mu \frac{d P}{d x} \quad \forall I^{* *}=\frac{b(2 h)^{3}}{12\left(1-v^{2}\right)}=\frac{2 b h^{3}}{3\left(1-v^{2}\right)} .
\end{aligned}
$$

By dropping the variable $b$, the formulated equation governing the static deflection takes the form

$$
\begin{array}{r}
D^{*} \frac{d^{4} W}{d x^{4}}+\frac{2 h}{\mu_{0}}\left(1-\frac{\mu_{0}}{\mu_{m}}\right) B_{0}{ }^{2} \frac{d^{2} W}{d x^{2}} \\
=h \mu \frac{d P}{d x} \quad \forall D=\frac{2 E h^{3}}{3\left(1-v^{2}\right)}
\end{array}
$$

On the other hand, we can rewrite (26b) to obtain the following specialized ordinary differential equations.

Case 3. A two-layer sandwich homogenous magnetoelastic beam-plate of thickness $h$ with non-uniform pressure at the interface. For such a problem, the formulated equation governing the static deflection takes the form

$$
\begin{gathered}
E I \frac{d^{4} W}{d x^{4}}+\frac{b h}{\mu_{0}}\left(1-\frac{\mu_{0}}{\mu_{m}}\right)\left(1-v^{2}\right) B_{0}{ }^{2} \frac{d^{2} W}{d x^{2}} \\
=\frac{1}{2} b h \mu\left(1-v^{2}\right) \frac{d P}{d x} \quad \forall I=\frac{b h^{3}}{12} .
\end{gathered}
$$

Case 4. A two-layer sandwich homogenous magnetoelastic beam-plate of thickness $2 h$ with non-uniform pressure at the interface. For such a problem, the formulated equation governing the static deflection takes the form

$$
\begin{array}{r}
E I^{*} \frac{d^{4} W}{d x^{4}}+\frac{2 b h}{\mu_{0}}\left(1-\frac{\mu_{0}}{\mu_{m}}\right)\left(1-v^{2}\right) B_{0}{ }^{2} \frac{d^{2} W}{d x^{2}} \\
=b h \mu\left(1-v^{2}\right) \frac{d P}{d x} \quad \forall I^{*}=\frac{2 E h^{3}}{3\left(1-v^{2}\right)}
\end{array}
$$

\section{Analysis of Static Deflection}

The generalized governing differential equation for the static deflection of each layer takes the following form:

$$
\frac{d^{4} W}{d x^{4}}+\frac{12}{\mu_{0}}\left(1-\frac{\mu_{0}}{\mu_{m}}\right) \frac{B_{0}^{2}}{E h^{2}} \frac{d^{2} W}{d x^{2}}=\alpha \frac{d P}{d x} ; \quad \forall \alpha=\frac{6 \mu}{E h^{2}} .
$$

For the cantilever architecture under investigation, the usual boundary conditions hold as follows:

$$
W(0)=\frac{d W(0)}{d x}=\frac{d^{2} W(L)}{d x^{2}}=0,
$$

in conjunction with the generalized end condition reported in Damisa et al. [19], namely,

$$
\int_{0}^{h} \tau_{(x z)_{1}}(x) d z=\frac{F}{2 b} \quad \text { at } x=L .
$$

By limiting our investigation to linear interface pressure profile, we obtain

$$
p(x)=p_{0}\left(1+\frac{\varepsilon}{L} x\right) .
$$

Equation (30) takes the form

$$
\frac{d^{4} W}{d x^{4}}+\frac{12}{\mu_{0}}\left(1-\frac{\mu_{0}}{\mu_{m}}\right) \frac{B_{0}^{2}}{E h^{2}} \frac{d^{2} W}{d x^{2}}=\alpha \frac{\varepsilon}{L} p_{0}
$$

The solution to the above is sorted via the Fourier finite sine transform namely,

$$
[\cdot]^{F}=\int_{0}^{L}[\cdot] \sin \left(\frac{n \pi x}{L}\right) d x ; \quad[\cdot]=\frac{2}{L} \sum_{n=0}^{\infty}[\cdot]^{F} \sin \left(\frac{n \pi x}{L}\right) .
$$

Equation (33) in the Fourier transform plane takes the form

$$
\begin{aligned}
\frac{n^{4} \pi^{4}}{L^{4}} & W^{F}\left(\lambda_{n}\right)-\frac{12 B_{0}^{2}}{E h^{2}}\left(\frac{1}{\mu_{0}}\left(1-\frac{\mu_{0}}{\mu_{m}}\right)\right) \frac{n^{2} \pi^{2}}{L^{2}} W^{F}\left(\lambda_{n}\right) \\
= & \left(\begin{array}{l}
\left(\frac{n^{3} \pi^{3}}{L^{3}}(-1)^{n+1}-\frac{12 B_{0}{ }^{2}}{E h^{2}}\left(\frac{1}{\mu_{0}}\left(1-\frac{\mu_{0}}{\mu_{m}}\right)\right) \frac{n \pi}{L}(-1)^{n+1}\right) W(L)-\frac{n \pi}{L} W_{x x}(0) \\
\alpha P_{0} \varepsilon\left(\frac{1+(-1)^{n+1}}{n \pi}\right)
\end{array}\right) .
\end{aligned}
$$


Following Damisa et al. [19], the bending moment and static deflection in the Fourier transform plane are computed as

$$
\begin{gathered}
W_{x x}(0)=\left\{\left(\frac{6 F}{E b h^{3}}-\frac{6 \mu P_{0}}{E h^{2}}\left(1+\frac{\varepsilon}{2}\right)\right) L+\frac{12 B_{0}^{2}}{E h^{2}} \frac{1}{\mu_{0}}\left(1-\frac{\mu_{0}}{\mu_{m}}\right) W(L)\right\}, \\
W^{F}\left(\lambda_{n}\right)=\frac{\left(\begin{array}{l}
\left(\frac{n^{3} \pi^{3}}{L^{3}}(-1)^{n+1}-\frac{n \pi}{L}(-1)^{n+1} \frac{12 B_{0}^{2}}{E h^{2}} \frac{1}{\mu_{0}}\left(1-\frac{\mu_{0}}{\mu_{m}}\right)\right) W(L) \\
-n \pi\left(\frac{6 F}{E b h^{3}}-\frac{6 \mu P_{0}\left(1+\frac{\varepsilon}{2}\right)}{E h^{2}}\right)-\frac{n \pi}{L} \frac{12 B_{0}^{2}}{E h^{2}} \frac{1}{\mu_{0}}\left(1-\frac{\mu_{0}}{\mu_{m}}\right) W(L)+\frac{6 \mu P_{0} \varepsilon}{E h^{2}}\left(\frac{1+(-1)^{n+1}}{n \pi}\right)
\end{array}\right)}{\left(\frac{n^{4} \pi^{4}}{L^{4}}-\frac{12 B_{0}^{2}}{E h^{2}}\left(\frac{1}{\mu_{0}}\left(1-\frac{\mu_{0}}{\mu_{m}}\right)\right) \frac{n^{2} \pi^{2}}{L^{2}}\right)} .
\end{gathered}
$$

The Fourier inversion of the above yields

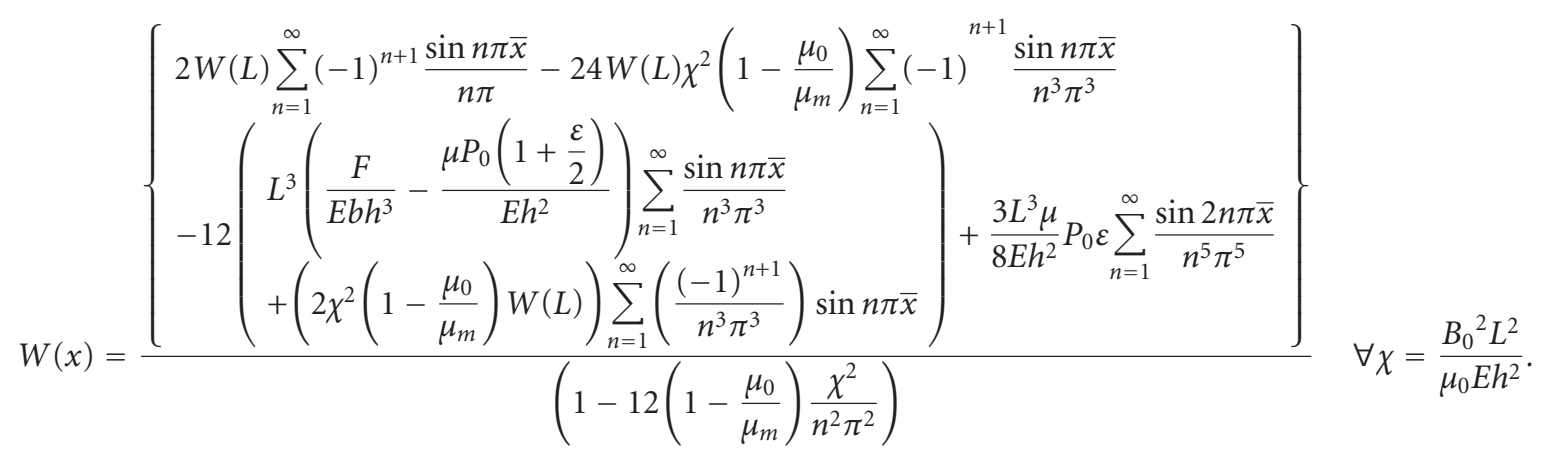

Utilizing binomial expansion, (37) is rewritten as

$$
\begin{aligned}
W(x)= & \left(1+12\left(1-\frac{\mu_{0}}{\mu_{m}}\right) \frac{\chi^{2}}{n^{2} \pi^{2}}\right) \\
& \times\left\{\begin{array}{l}
2 W(L) \sum_{n=1}^{\infty}(-1)^{n+1} \frac{\sin n \pi \bar{x}}{n \pi}-24 W(L) \chi^{2}\left(1-\frac{\mu_{0}}{\mu_{m}}\right) \sum_{n=1}^{\infty}(-1)^{n+1} \frac{\sin n \pi \bar{x}}{n^{3} \pi^{3}} \\
-12\left(\begin{array}{l}
L^{3}\left(\frac{F}{E b h^{3}}-\frac{\mu P_{0}\left(1+\frac{\varepsilon}{2}\right)}{E h^{2}}\right) \sum_{n=1}^{\infty} \frac{\sin n \pi \bar{x}}{n^{3} \pi^{3}} \\
+2 \chi^{2}\left(1-\frac{\mu_{0}}{\mu_{m}}\right) W(L) \sum_{n=1}^{\infty} \frac{\sin n \pi \bar{x}}{n^{3} \pi^{3}}
\end{array}\right)+\frac{3 L^{3} \mu}{8 E h^{2}} P_{0} \varepsilon \sum_{n=1}^{\infty} \frac{\sin 2 n \pi \bar{x}}{n^{5} \pi^{5}}
\end{array}\right\} .
\end{aligned}
$$


The semiinfinite series in (38) can be converted to spatial polynomials via the following closed form Fourier series representations:

$$
\begin{gathered}
\bar{x}=\frac{1}{\pi} \sum_{n=1}^{\infty} \frac{(-1)^{n+1}}{n} \sin n \pi \bar{x}, \\
\forall 0<\bar{x}<1, \\
\sum_{n=1}^{\infty} \frac{\sin n \bar{x}}{n^{3}}=\frac{\pi^{2} \bar{x}}{6}-\frac{\pi \bar{x}^{2}}{4}+\frac{\bar{x}^{3}}{12}, \\
\forall 0<\bar{x}<2,
\end{gathered}
$$

$$
\sum_{n=1}^{\infty} \frac{\sin n \bar{x}}{n^{5}}=\frac{\pi^{4} \bar{x}}{90}-\frac{\pi^{2} \bar{x}^{3}}{36}+\frac{\pi \bar{x}^{4}}{48}-\frac{\bar{x}^{5}}{240},
$$$$
\forall 0<\bar{x}<2 \text {, }
$$$$
\sum_{n=1}^{\infty} \frac{\sin n \bar{x}}{n^{7}}=\frac{2 \pi^{6} \bar{x}}{405}-\frac{\pi^{4} \bar{x}^{3}}{540}+\frac{\pi^{2} \bar{x}^{5}}{720}-\frac{\pi \bar{x}^{6}}{1440}+\frac{\bar{x}^{7}}{980},
$$

$\forall 0<\bar{x}<2$.

Consequently, we can write (39) in the form

$$
W(x)=\left\{\begin{array}{l}
2 W(L) \bar{x}-12 L^{3}\left(\left(\frac{F}{E b h^{3}}-\frac{\mu P_{0}(1+\varepsilon / 2)}{E h^{2}}\right) \Lambda_{1}\right)+\frac{3 L^{3} \mu}{8 E h^{2}} P_{0} \varepsilon \Lambda_{2}-W(L)\left(24 \chi^{2}\left(1-\frac{\mu_{0}}{\mu_{m}}\right)+15 \chi^{4}\left(1-\frac{\mu_{0}}{\mu_{m}}\right)^{2}\right) \Lambda_{2} \\
-144 L^{3} \chi^{2}\left(1-\frac{\mu_{0}}{\mu_{m}}\right)\left(\frac{F}{E b h^{3}}-\frac{\mu P_{0}(1+\varepsilon / 2)}{E h^{2}}\right) \Lambda_{3}+\frac{9 L^{3} \mu}{2 E h^{2}} \chi^{2}\left(1-\frac{\mu_{0}}{\mu_{m}}\right) P_{0} \varepsilon \Lambda_{4}
\end{array}\right\},
$$

where

$$
\begin{aligned}
& \Lambda_{1}=\left(\begin{array}{ll}
\left.\frac{\bar{x}}{6}-\frac{\bar{x}^{2}}{4}+\frac{\bar{x}^{3}}{12}\right) ; & \Lambda_{4}=\frac{4 \bar{x}}{405}-\frac{2 \bar{x}^{3}}{135}+\frac{2 \bar{x}^{5}}{45}-\frac{2 \bar{x}^{6}}{45}+\frac{64 \bar{x}^{7}}{245} . \\
\Lambda_{2}=\left(\frac{\bar{x}}{45}-\frac{2 \bar{x}^{3}}{9}+\frac{\bar{x}^{4}}{3}-\frac{2 \bar{x}^{5}}{5}\right) ; & \begin{array}{l}
\text { Imposing the condition } d \bar{W} / d x=0 \text { in (40), the deflection at } \\
\text { the end of the sandwich magnetoelastic cantilever structure }
\end{array} \\
\Lambda_{3}=\left(\frac{\bar{x}}{90}-\frac{\bar{x}^{3}}{36}+\frac{\bar{x}^{4}}{48}-\frac{\bar{x}^{5}}{240}\right) ; & \text { is } \\
W(L)=\left(\begin{array}{ll}
\left(\frac{F}{E b h^{3}}-\frac{\mu P_{0}(1+\varepsilon / 2)}{E h^{2}}-\frac{\mu P_{0} \varepsilon}{240 E h^{2}}\right)+\chi^{2}\left(1-\frac{\mu_{0}}{\mu_{m}}\right)\left(\frac{F}{E b h^{3}}-\frac{\mu P_{0}}{E h^{2}}\right) \\
+\frac{13}{50} \chi^{4}\left(1-\frac{\mu_{0}}{\mu_{m}}\right)^{2}\left(\frac{F}{E b h^{3}}-\frac{\mu P_{0}(1+\varepsilon / 2)}{E h^{2}}-\frac{5 \mu P_{0} \varepsilon}{26 E h^{2}}\right)+\frac{2}{25} \chi^{6}\left(1-\frac{\mu_{0}}{\mu_{m}}\right)^{3}\left(\frac{F}{E b h^{3}}-\frac{\mu P_{0}}{E h^{2}}\right)
\end{array}\right) .
\end{array}\right.
\end{aligned}
$$

Substitution of the above with rearrangement gives

$$
\bar{W}(\bar{x})=\left\{\begin{array}{l}
\left(1-\mu \bar{P}_{0}\right)\left(3 \bar{x}^{2}-\bar{x}^{3}\right)+\mu \bar{P}_{0} \varepsilon\left(-3 \bar{x}^{2}+\frac{11}{12} \bar{x}^{3}+\frac{1}{8} \bar{x}^{4}-\frac{1}{20} \bar{x}^{5}\right) \\
+\chi^{2}\left(1-\frac{\mu_{0}}{\mu_{m}}\right)\left(\left(1-\mu \bar{P}_{0}\right)\left(-\frac{28}{13} \bar{x}^{3}+11 \bar{x}^{4}+\frac{51}{5} \bar{x}^{5}\right)+\mu \bar{P}_{0} \varepsilon\left(-\frac{214}{45} \bar{x}^{3}-\frac{83}{15} \bar{x}^{4}+\frac{267}{50} \bar{x}^{5}-\frac{1}{5} \bar{x}^{6}+\frac{288}{245} \bar{x}^{8}\right)\right) \\
+\chi^{4}\left(1-\frac{\mu_{0}}{\mu_{m}}\right)^{2}\left(\left(1-\mu \bar{P}_{0}\right)\left(\frac{26}{3} \bar{x}^{3}-13 \bar{x}^{4}+\frac{78}{5} \bar{x}^{5}\right)+\mu \bar{P}_{0} \varepsilon\left(-\frac{121}{72} \bar{x}^{3}+\frac{121}{48} \bar{x}^{4}+\frac{121}{40} \bar{x}^{5}\right)\right) \\
+\chi^{6}\left(1-\frac{\mu_{0}}{\mu_{m}}\right)^{3}\left(\left(1-\mu \bar{P}_{0}\right)\left(\frac{-354}{75} \bar{x}^{3}-\frac{177}{25} \bar{x}^{4}+\frac{1107}{125} \bar{x}^{5}\right)+\mu \bar{P}_{0} \varepsilon\left(-\frac{1}{20} \bar{x}^{3}+\frac{20}{13} \bar{x}^{4}-\frac{24}{13} \bar{x}^{5}\right)\right) \\
+\chi^{8}\left(1-\frac{\mu_{0}}{\mu_{m}}\right)^{4}\left(\left(1-\mu \bar{P}_{0}\right)\left(\frac{97}{75} \bar{x}^{3}-\frac{97}{50} \bar{x}^{4}+\frac{296}{125} \bar{x}^{5}\right)+\mu \bar{P}_{0} \varepsilon\left(-\frac{1}{6} \bar{x}^{3}-\frac{20}{13} \bar{x}^{4}+\frac{33}{50} \bar{x}^{5}\right)\right) \\
+\chi^{10}\left(1-\frac{\mu_{0}}{\mu_{m}}\right)^{5}\left(\left(1-\mu \bar{P}_{0}\right)\left(\frac{4}{15} \bar{x}^{3}-\frac{2}{5} \bar{x}^{4}+\frac{12}{25} \bar{x}^{5}\right)\right)
\end{array}\right\},
$$


where the following nondimensionalized parameters have been introduced as

$$
\bar{W}(\bar{x})=\frac{W(\bar{x}) E b h^{3}}{L^{3} F} ; \quad \bar{P}_{0}=\frac{P_{0}}{(F / b h)} ; \quad \bar{x}=\frac{x}{L}
$$

For the special case $\varepsilon \rightarrow 0$ in (43), the transverse deflection at uniform pressure is

$$
\begin{aligned}
& \bar{W}(\bar{x}) \\
& =\left\{\begin{array}{l}
\left(1-\mu \bar{P}_{0}\right)\left(3 \bar{x}^{2}-\bar{x}^{3}\right) \\
+\chi^{2}\left(1-\frac{\mu_{0}}{\mu_{m}}\right)\left(\left(1-\mu \bar{P}_{0}\right)\left(-\frac{28}{13} \bar{x}^{3}+11 \bar{x}^{4}+\frac{51}{5} \bar{x}^{5}\right)\right) \\
+\chi^{4}\left(1-\frac{\mu_{0}}{\mu_{m}}\right)^{2}\left(\left(1-\mu \bar{P}_{0}\right)\left(\frac{26}{3} \bar{x}^{3}-13 \bar{x}^{4}+\frac{78}{5} \bar{x}^{5}\right)\right) \\
+\chi^{6}\left(1-\frac{\mu_{0}}{\mu_{m}}\right)^{3}\left(\left(1-\mu \bar{P}_{0}\right)\left(\frac{-354}{75} \bar{x}^{3}-\frac{177}{25} \bar{x}^{4}+\frac{1107}{125} \bar{x}^{5}\right)\right) \\
+\chi^{8}\left(1-\frac{\mu_{0}}{\mu_{m}}\right)^{4}\left(\left(1-\mu \bar{P}_{0}\right)\left(\frac{97}{75} \bar{x}^{3}-\frac{97}{50} \bar{x}^{4}+\frac{296}{125} \bar{x}^{5}\right)\right) \\
+\chi^{10}\left(1-\frac{\mu_{0}}{\mu_{m}}\right)^{5}\left(\left(1-\mu \bar{P}_{0}\right)\left(\frac{4}{15} \bar{x}^{3}-\frac{2}{5} \bar{x}^{4}+\frac{12}{25} \bar{x}^{5}\right)\right)
\end{array}\right\},
\end{aligned}
$$

which for the case of $\chi=0$ agrees with the results in Damisa et al. [19].

\section{Analysis of Static Slip}

As shown in Figure 1(e), during bending, each half of the layered elastic structure has its own neutral plane that does not necessarily coincide with the geometric mid plane through the interface because of the frictional stresses. For the sandwich structure, the geometrical description of the gross interfacial slip is defined in Figure 1(e). In view of the foregoing, the expressions for the displacements of the two adjacent opposite points follow from Taylor series approximation as:

$$
\begin{aligned}
& \Delta U_{1}(x, z) \\
& =\left(\Delta U_{1}(0,0+)+\left(z-\psi(x) \frac{h}{2}\right) \frac{d W(x)}{d x}+\frac{1}{2}\left(z-\psi(x) \frac{h}{2}\right)^{2}\right. \\
& \left.\quad \times \frac{d^{2} W(x)}{d x^{2}}+\frac{1}{6}\left(z-\psi(x) \frac{h}{2}\right)^{3} \frac{d^{3} W(x)}{d x^{3}}\right),
\end{aligned}
$$

$$
\Delta U_{2}(x, z)
$$

$$
\begin{gathered}
=\left(\Delta U_{2}(0,0-)+\left(z+\psi(x) \frac{h}{2}\right) \frac{d W(x)}{d x}+\frac{1}{2}\left(z+\psi(x) \frac{h}{2}\right)^{2}\right. \\
\left.\times \frac{d^{2} W(x)}{d x^{2}}+\frac{1}{6}\left(z+\psi(x) \frac{h}{2}\right)^{3} \frac{d^{3} W(x)}{d x^{3}}\right)
\end{gathered}
$$

which for the case of first order theory reduce to the forms

$$
\begin{aligned}
& \Delta U_{1}(x, z)=\Delta U_{1}(0,0+)+\left(z-\psi(x) \frac{h}{2}\right) \frac{d W(x)}{d x} \\
& \Delta U_{2}(x, z)=\Delta U_{2}(0,0-)+\left(z+\psi(x) \frac{h}{2}\right) \frac{d W(x)}{d x}
\end{aligned}
$$

For this problem, $\Delta u_{1}(0,0+)$ and $\Delta u_{2}(0,0-)$ must be zero at the fixed end. Hence, the relative static slip at the interface of the elastic structure is given by

$$
\Delta U(x, 0)=\Delta U_{1}(x, 0+)-\Delta U_{2}(x, 0-) .
$$

Following Goodman and Klumpp [24], (49) becomes

$$
\Delta U(x, 0)=E^{-1} \int_{0}^{x}\left\{\left(\sigma_{x}\right)_{1}(\xi, 0+)-\left(\sigma_{x}\right)_{2}(\xi, 0-)\right\} d \xi
$$

where $\xi$ is a dummy axial spatial variable of integration across the interface, and $0+, 0-$ denote the origin of the transverse spatial variable for each layer; where subscripts 1 and 2 refer to the upper and lower laminates.

On substituting (23)-(24) into the first parts of (15a)(15b), the derived corresponding spatial bending stresses are, namely,

$$
\begin{aligned}
\left(\sigma_{x}\right)_{1}(x, z) & \\
= & -\frac{E}{2}(2 z-h) \frac{d^{2} W(x)}{d x^{2}}+\frac{B_{0}^{2}}{\mu_{0}}\left(1-\frac{\mu_{0}}{\mu_{m}}\right) \\
& \times(W(x)-W(L))+\mu P_{0}(1+\varepsilon \bar{x}) \frac{(x-L)}{h}, \\
\left(\sigma_{x}\right)_{2}(x, z) & \\
= & -\frac{E}{2}(2 z+h) \frac{d^{2} W(x)}{d x^{2}}-\frac{B_{0}^{2}}{\mu_{0}}\left(1-\frac{\mu_{0}}{\mu_{m}}\right) \\
& \times(W(x)-W(L))-\mu P_{0}(1+\varepsilon \bar{x}) \frac{(x-L)}{h} .
\end{aligned}
$$

This gives (50) as

$$
\begin{aligned}
\Delta U(\bar{x}) & =\int_{0}^{\bar{x}}\left\{\frac{d^{2} \bar{W}(\bar{x})}{d \bar{\xi}^{2}}+2 \bar{B}_{0}^{2}\left(1-\frac{\mu_{0}}{\mu_{m}}\right)\right. \\
& \left.\times(\bar{W}(\bar{\xi})-\bar{W}(1))+2 \mu \bar{P}_{0}(1+\varepsilon \bar{\xi})(\bar{\xi}-1)\right\} d \bar{\xi},
\end{aligned}
$$

and on introducing the following nondimensionalized parameters:

$$
\Delta \bar{U}=\frac{\Delta U(\bar{x}) E b h^{2}}{L^{2} F} ; \quad \bar{P}_{0}=\frac{P_{0}}{(F / b h)} .
$$


Equation (52) simplifies to the form

$$
\Delta \bar{U}=\left\{\begin{array}{l}
\left(4 \mu \bar{P}_{0}-3\right)\left(\bar{x}^{2}-2 \bar{x}\right)+\mu \bar{P}_{0} \varepsilon\left(-6 \bar{x}+\frac{7 \bar{x}^{2}}{4}+\frac{7 \bar{x}^{3}}{6}-\frac{\bar{x}^{4}}{4}\right) \\
+\chi^{2}\left(1-\frac{\mu_{0}}{\mu_{m}}\right)\left(\left(1-\mu \bar{P}_{0}\right) \Pi_{1}(\bar{x})+\mu \bar{P}_{0} \varepsilon \Pi_{2}(\bar{x})\right)+\chi^{4}\left(1-\frac{\mu_{0}}{\mu_{m}}\right)^{2}\left(\left(1-\mu \bar{P}_{0}\right) \Pi_{3}(\bar{x})+\mu \bar{P}_{0} \varepsilon \Pi_{4}(\bar{x})\right) \\
+\chi^{6}\left(1-\frac{\mu_{0}}{\mu_{m}}\right)^{3}\left(\left(1-\mu \bar{P}_{0}\right) \Pi_{5}(\bar{x})+\mu \bar{P}_{0} \varepsilon \Pi_{6}(\bar{x})\right)+\chi^{8}\left(1-\frac{\mu_{0}}{\mu_{m}}\right)^{4}\left(\left(1-\mu \bar{P}_{0}\right) \Pi_{7}(\bar{x})+\mu \bar{P}_{0} \varepsilon \Pi_{8}(\bar{x})\right) \\
+\chi^{10}\left(1-\frac{\mu_{0}}{\mu_{m}}\right)^{5}\left(\left(1-\mu \bar{P}_{0}\right) \Pi_{9}(\bar{x})+\mu \bar{P}_{0} \varepsilon \Pi_{10}(\bar{x})\right)+\chi^{12}\left(1-\frac{\mu_{0}}{\mu_{m}}\right)^{6}\left(1-\mu \bar{P}_{0}\right) \Pi_{11}(\bar{x})
\end{array}\right\},
$$

where

$$
\begin{aligned}
\Pi_{1}(\bar{x})= & \left(2 \bar{x}-\frac{84}{13} \bar{x}^{2}+44 \bar{x}^{3}+51 \bar{x}^{4}\right), \\
\Pi_{2}(\bar{x})=( & -\frac{241}{120} \bar{x}-\frac{214}{15} \bar{x}^{2}-\frac{332}{15} \bar{x}^{3}+\frac{6463}{240} \bar{x}^{4} \\
& \left.-\frac{47}{40} \bar{x}^{5}-\frac{1}{120} \bar{x}^{6}-\frac{2304}{245} \bar{x}^{7}\right), \\
\Pi_{3}(\bar{x})= & \left(\frac{1283}{65} \bar{x}+\frac{1}{26} \bar{x}^{2}-52 \bar{x}^{3}-\frac{1021}{13} \bar{x}^{4}+\frac{11}{5} \bar{x}^{5}+\frac{17}{10} \bar{x}^{6}\right), \\
\Pi_{4}(\bar{x})= & \left(4 \bar{x}-\frac{121}{24} \bar{x}^{2}+\frac{121}{12} \bar{x}^{3}+\frac{5017}{360} \bar{x}^{4}\right. \\
& \left.-\frac{83}{75} \bar{x}^{5}+\frac{267}{300} \bar{x}^{6}-\frac{1}{35} \bar{x}^{7}+\frac{32}{245} \bar{x}^{9}\right), \\
\Pi_{5}(\bar{x})= & \left(\frac{169}{5} \bar{x}-\frac{354}{25} \bar{x}^{2}-\frac{708}{25} \bar{x}^{3}+\frac{6967}{150} \bar{x}^{4}-\frac{13}{5} \bar{x}^{5}+\frac{39}{15} \bar{x}^{6}\right), \\
\Pi_{6}(\bar{x})= & \left(\frac{2783}{720} \bar{x}-\frac{3}{20} \bar{x}^{2}+\frac{80}{13} \bar{x}^{3}\right. \\
& \left.-\frac{36133}{3744} \bar{x}^{4}+\frac{121}{240} \bar{x}^{5}+\frac{121}{240} \bar{x}^{6}\right),
\end{aligned}
$$$$
\Pi_{7}(\bar{x})=\left(-\frac{368}{125} \bar{x}-\frac{97}{25} \bar{x}^{2}-\frac{194}{25} \bar{x}^{3}\right.
$$$$
\left.+\frac{533}{50} \bar{x}^{4}-\frac{177}{125} \bar{x}^{5}+\frac{1107}{750} \bar{x}^{6}\right),
$$$$
\Pi_{8}(\bar{x})=\left(\frac{-93}{260} \bar{x}-\frac{1}{2} \bar{x}^{2}+\frac{80}{13} \bar{x}^{3}-\frac{53}{16} \bar{x}^{4}+\frac{4}{13} \bar{x}^{5}-\frac{4}{13} \bar{x}^{6}\right),
$$$$
\Pi_{9}(\bar{x})=\left(\frac{1291}{750} \bar{x}-\frac{97}{25} \bar{x}^{2}-\frac{194}{25} \bar{x}^{3}\right.
$$$$
\left.+\frac{3649}{300} \bar{x}^{4}+\frac{97}{250} \bar{x}^{5}-\frac{148}{375} \bar{x}^{6}\right),
$$$$
\Pi_{10}(\bar{x})=\left(\frac{-1019}{975} \bar{x}-\frac{1}{2} \bar{x}^{2}+\frac{80}{13} \bar{x}^{3}-\frac{401}{120} \bar{x}^{4}-\frac{4}{13} \bar{x}^{5}+\frac{11}{100} \bar{x}^{6}\right),
$$$$
\Pi_{11}(\bar{x})=\left(\frac{26}{75} \bar{x}+\frac{1}{15} \bar{x}^{4}-\frac{1}{5} \bar{x}^{5}+\frac{2}{25} \bar{x}^{6}\right) .
$$

$$
\Delta \bar{U}=\left\{\begin{array}{l}
\left(4 \mu \bar{P}_{0}-3\right)\left(\bar{x}^{2}-2 \bar{x}\right)+\chi^{2}\left(1-\frac{\mu_{0}}{\mu_{m}}\right)\left(1-\mu \bar{P}_{0}\right) \Pi_{1}(\bar{x})+\chi^{4}\left(1-\frac{\mu_{0}}{\mu_{m}}\right)^{2}\left(1-\mu \bar{P}_{0}\right) \Pi_{3}(\bar{x}) \\
+\chi^{6}\left(1-\frac{\mu_{0}}{\mu_{m}}\right)^{3}\left(1-\mu \bar{P}_{0}\right) \Pi_{5}(\bar{x})+\chi^{8}\left(1-\frac{\mu_{0}}{\mu_{m}}\right)^{4}\left(1-\mu \bar{P}_{0}\right) \Pi_{7}(\bar{x}) \\
+\chi^{10}\left(1-\frac{\mu_{0}}{\mu_{m}}\right)^{5}\left(1-\mu \bar{P}_{0}\right) \Pi_{9}(\bar{x})+\chi^{12}\left(1-\frac{\mu_{0}}{\mu_{m}}\right)^{6}\left(1-\mu \bar{P}_{0}\right) \Pi_{11}(\bar{x})
\end{array}\right\} .
$$


The case $\chi=0$ agrees with the result in [19]. The foregoing expression allows us to compute the maximum slip at the tip of the magnetoelastic structure in the context of the pressure gradient as

$$
\Delta \bar{U}=\left\{\begin{array}{l}
\left(3-4 \mu \bar{P}_{0}\right)-3.33 \mu \bar{P}_{0} \varepsilon+\chi^{2}\left(1-\frac{\mu_{0}}{\mu_{m}}\right)\left(91\left(1-\mu \bar{P}_{0}\right)-24 \mu \bar{P}_{0} \varepsilon\right) \\
+\chi^{4}\left(1-\frac{\mu_{0}}{\mu_{m}}\right)^{2}\left(-107\left(1-\mu \bar{P}_{0}\right)-22.9 \mu \bar{P}_{0} \varepsilon\right)+\chi^{6}\left(1-\frac{\mu_{0}}{\mu_{m}}\right)^{3}\left(38\left(1-\mu \bar{P}_{0}\right)+1.23 \mu \bar{P}_{0} \varepsilon\right) \\
+\chi^{8}\left(1-\frac{\mu_{0}}{\mu_{m}}\right)^{4}\left(-3.86\left(1-\mu \bar{P}_{0}\right)+1.98 \mu \bar{P}_{0} \varepsilon\right)+\chi^{10}\left(1-\frac{\mu_{0}}{\mu_{m}}\right)^{5}\left(2.24\left(1-\mu \bar{P}_{0}\right)+1.07 \mu \bar{P}_{0} \varepsilon\right) \\
-0.3 \chi^{12}\left(1-\frac{\mu_{0}}{\mu_{m}}\right)^{6}\left(1-\mu \bar{P}_{0}\right)
\end{array}\right\} .
$$

On setting $\varepsilon \rightarrow 0$ in (57), the maximum static slip at the tip for the case of uniform pressure is

$$
\Delta \bar{U}_{\max (\text { tip })}=\left\{\begin{array}{l}
\left(3-4 \mu \bar{P}_{0}\right)+91 \chi^{2}\left(1-\frac{\mu_{0}}{\mu_{m}}\right)\left(1-\mu \bar{P}_{0}\right)-107 \chi^{4}\left(1-\frac{\mu_{0}}{\mu_{m}}\right)^{2}\left(1-\mu \bar{P}_{0}\right) \\
+38 \chi^{6}\left(1-\frac{\mu_{0}}{\mu_{m}}\right)^{3}\left(1-\mu \bar{P}_{0}\right)-3.86 \chi^{8}\left(1-\frac{\mu_{0}}{\mu_{m}}\right)^{4}\left(1-\mu \bar{P}_{0}\right) \\
+2.24 \chi^{10}\left(1-\frac{\mu_{0}}{\mu_{m}}\right)^{5}\left(1-\mu \bar{P}_{0}\right)-0.3 \chi^{12}\left(1-\frac{\mu_{0}}{\mu_{m}}\right)^{6}\left(1-\mu \bar{P}_{0}\right)
\end{array}\right\} .
$$

This suggests that in the presence of coulomb friction and interfacial pressure $\bar{P}_{0}$, there are critical values of pressure for which no slip occurs at the tip such as

$$
\bar{P}_{0(\text { critical })}=0.75 \mu^{-1} \frac{\Upsilon_{1}\left(\chi, \bar{B}_{0}\right)}{\Upsilon_{2}\left(\chi, \bar{B}_{0}\right)},
$$

$$
\begin{aligned}
& \Upsilon_{1}\left(\chi, \bar{B}_{0}\right)=\left(\begin{array}{l}
1+\frac{91}{3} \chi^{2}\left(1-\frac{\mu_{0}}{\mu_{m}}\right)-\frac{107}{3} \bar{B}_{0}^{4}\left(1-\frac{\mu_{0}}{\mu_{m}}\right)^{2}+\frac{38}{3} \chi^{6}\left(1-\frac{\mu_{0}}{\mu_{m}}\right)^{3}-\frac{3.86}{3} \chi^{8}\left(1-\frac{\mu_{0}}{\mu_{m}}\right)^{4} \\
+\frac{2.24}{3} \chi^{10}\left(1-\frac{\mu_{0}}{\mu_{m}}\right)^{5}-\frac{1}{10} \chi^{12}\left(1-\frac{\mu_{0}}{\mu_{m}}\right)^{6}
\end{array}\right) \\
& \Upsilon_{2}\left(\chi, \bar{B}_{0}\right)=\left(\begin{array}{l}
1+\frac{3.33}{4}+\chi^{2}\left(1-\frac{\mu_{0}}{\mu_{m}}\right)\left(\frac{91}{4}+6 \varepsilon\right)-\chi^{4}\left(1-\frac{\mu_{0}}{\mu_{m}}\right)^{2}\left(\frac{107}{4}-\frac{22.9}{4} \varepsilon\right) \\
+\chi^{6}\left(1-\frac{\mu_{0}}{\mu_{m}}\right)^{3}\left(\frac{38}{4}-\frac{1.23}{4} \varepsilon\right)-\chi^{8}\left(1-\frac{\mu_{0}}{\mu_{m}}\right)^{4}\left(\frac{3.86}{4}+\frac{1.23}{4} \varepsilon\right) \\
+\chi^{10}\left(1-\frac{\mu_{0}}{\mu_{m}}\right)^{5}\left(\frac{2.24}{4}-\frac{1.07}{4} \varepsilon\right)-\frac{3}{10} \chi^{12}\left(1-\frac{\mu_{0}}{\mu_{m}}\right)^{5}
\end{array}\right) .
\end{aligned}
$$


The case $\varepsilon \rightarrow 0$ gives the expression for critical values of pres- where sure for which no slip occurs at the tip as

$$
\bar{P}_{0(\text { critical })}=0.75 \mu^{-1} \frac{\Upsilon_{1}\left(\chi, \bar{B}_{0}\right)}{\Upsilon_{3}\left(\chi, \bar{B}_{0}\right)},
$$

$$
\Upsilon_{3}\left(\chi, \bar{B}_{0}\right)=\left(\begin{array}{l}
1+\frac{3.33}{4}+\frac{91}{4} \chi^{2}\left(1-\frac{\mu_{0}}{\mu_{m}}\right)-\frac{107}{4} \chi^{4}\left(1-\frac{\mu_{0}}{\mu_{m}}\right)^{2}+\frac{38}{4} \chi^{6}\left(1-\frac{\mu_{0}}{\mu_{m}}\right)^{3}-\frac{3.86}{4} \chi^{8}\left(1-\frac{\mu_{0}}{\mu_{m}}\right)^{4} \\
+\frac{2.24}{4} \chi^{10}\left(1-\frac{\mu_{0}}{\mu_{m}}\right)^{5}-\frac{3}{10} \chi^{12}\left(1-\frac{\mu_{0}}{\mu_{m}}\right)^{5}
\end{array}\right)
$$

which for the case of $\bar{B}_{0}=0$ agrees with the result in [19].

which can also be expressed as

\section{Energy Dissipation}

The energy dissipated per static slip, following Damisa [25] is given by the relation

$$
\bar{D}=4 \mu \bar{p}_{0} \int_{0}^{1}\left(1+\frac{\varepsilon}{2}\right) \Delta \bar{u} d \bar{x} \quad \forall \bar{p}_{\mathrm{av}}=\bar{p}_{0} \int_{0}^{1}(1+\varepsilon x) d \bar{x}
$$

$$
D=4 \mu b \int_{0}^{L} p(x) \Delta u(x) d x
$$

on substituting for $\Delta \bar{u}$ gives

$$
\bar{D}=\left(\begin{array}{l}
\left(8 \mu \bar{p}_{0}-\frac{32}{3} \mu^{2} \bar{p}_{0}{ }^{2}\right)+4 \mu \bar{p}_{0} \varepsilon-\frac{421}{30} \mu^{2} \bar{p}_{0}{ }^{2} \varepsilon-\frac{261}{60} \mu^{2} \bar{p}_{0}{ }^{2} \varepsilon^{2} \\
+\chi^{2}\left(1-\frac{\mu_{0}}{\mu_{m}}\right)\left(80 \mu \bar{p}_{0}-80 \mu^{2} \bar{p}_{0}{ }^{2}+20 \mu \bar{p}_{0} \varepsilon-69.2 \mu^{2} \bar{p}_{0}{ }^{2} \varepsilon-\frac{29.2}{2} \mu^{2} \bar{p}_{0}{ }^{2} \varepsilon^{2}\right) \\
+\chi^{4}\left(1-\frac{\mu_{0}}{\mu_{m}}\right)^{2}\left(-72.8 \mu \bar{p}_{0}+72.8 \mu^{2} \bar{p}_{0}{ }^{2}-36.4 \mu \bar{p}_{0} \varepsilon+14 \mu^{2} \bar{p}_{0}{ }^{2} \varepsilon-11.2 \mu^{2} \bar{p}_{0}{ }^{2} \varepsilon^{2}\right) \\
+\chi^{8}\left(1-\frac{\mu_{0}}{\mu_{m}}\right)^{3}\left(57.2 \mu \bar{p}_{0}-57.2 \mu^{2} \bar{p}_{0}{ }^{2}+28.6 \mu \bar{p}_{0} \varepsilon-21.8 \mu^{2} \bar{p}_{0}{ }^{2} \varepsilon+3.4 \mu^{2} \bar{p}_{0}{ }^{2} \varepsilon^{2}\right) \\
+\chi^{10}\left(1-\frac{\mu_{0}}{\mu_{m}}\right)^{5}\left(0.28 \mu \bar{p}_{0}-0.28 \mu^{2} \bar{p}_{0}{ }^{2}+0.14 \mu \bar{p}_{0} \varepsilon-0.46 \mu^{2} \bar{p}_{0}{ }^{2} \varepsilon+0.3 \mu^{2} \bar{p}_{0}{ }^{2} \varepsilon^{2}\right) \\
+\chi^{12}\left(1-\frac{\mu_{0}}{\mu_{m}}\right)^{6}\left(0.68 \mu \bar{p}_{0}-0.68 \mu^{2} \bar{p}_{0}{ }^{2}+0.32 \mu \bar{p}_{0} \varepsilon-0.32 \mu^{2} \bar{p}_{0}{ }^{2} \varepsilon^{2}\right)
\end{array}\right),
$$

where $\bar{D}=D E b h^{3} /\left(L^{3} F_{0}^{2}\right)$ is the dimensionless static energy dissipated.

5.1. Analysis of Optimum Clamping Pressure. The optimum clamping pressure can be found from the partial derivative of the energy dissipated if we set

$$
\frac{\partial \bar{D}}{\partial \bar{p}_{0}}=0
$$

Thus we can derive the general expression for the optimum clamping pressure as

$$
\bar{p}_{\mathrm{opt}}=0.375 \mu^{-1} \frac{\Lambda_{1}}{\Lambda_{2}}
$$


where

$$
\begin{aligned}
& \Lambda_{1}=\left(\begin{array}{l}
1+\frac{1}{2} \varepsilon+\frac{5}{2} \chi^{2}\left(1-\frac{\mu_{0}}{\mu_{m}}\right)(1+4 \varepsilon)+\frac{91}{20} \chi^{4}\left(1-\frac{\mu_{0}}{\mu_{m}}\right)^{2}(2-\varepsilon)+\frac{143}{40} \chi^{6}\left(1-\frac{\mu_{0}}{\mu_{m}}\right)^{3}(2+\varepsilon) \\
+\frac{13}{20} \chi^{8}\left(1-\frac{\mu_{0}}{\mu_{m}}\right)^{4}(-2-\varepsilon)+\frac{7}{400} \chi^{10}\left(1-\frac{\mu_{0}}{\mu_{m}}\right)^{5}(2+\varepsilon)+\frac{17}{12} \chi^{12}\left(1-\frac{\mu_{0}}{\mu_{m}}\right)^{6}(1+\varepsilon)
\end{array}\right) \\
& \Lambda_{2}=\left(\begin{array}{l}
1+\frac{421}{320} \varepsilon+\frac{261}{640} \varepsilon^{2}+\chi^{2}\left(1-\frac{\mu_{0}}{\mu_{m}}\right)\left(\frac{15}{2}+\frac{81}{25} \varepsilon+\frac{137}{100} \varepsilon^{2}\right) \\
+\chi^{4}\left(1-\frac{\mu_{0}}{\mu_{m}}\right)^{2}\left(-\frac{683}{100}-\frac{131}{100} \varepsilon+\frac{21}{20} \varepsilon^{2}\right)+\chi^{6}\left(1-\frac{\mu_{0}}{\mu_{m}}\right)^{3}\left(\frac{107}{20}+\frac{51}{20} \varepsilon-\frac{8}{25} \varepsilon^{2}\right) \\
+\chi^{8}\left(1-\frac{\mu_{0}}{\mu_{m}}\right)^{4}\left(-\frac{49}{50}-\frac{69}{100} \varepsilon-\frac{103}{1000} \varepsilon^{2}\right)+\chi^{10}\left(1-\frac{\mu_{0}}{\mu_{m}}\right)^{5}\left(\frac{3}{100}+\frac{43}{1000} \varepsilon-\frac{3}{100} \varepsilon^{2}\right) \\
+\chi^{12}\left(1-\frac{\mu_{0}}{\mu_{m}}\right)^{6}\left(\frac{16}{25}+\frac{3}{100} \varepsilon\right)
\end{array}\right.
\end{aligned}
$$

In the limit as $\chi \rightarrow 0$, we recover the optimum clamping pressure in [19] as

$$
\widehat{\bar{p}}_{0}=0.375 \mu^{-1}\left\{\left(1+\frac{1}{2} \varepsilon\right)\left(1+\frac{421}{320} \varepsilon+\frac{261}{640} \varepsilon^{2}\right)^{-1}\right\} .
$$

By letting $\varepsilon \rightarrow 0$, the optimum clamping pressure for the magnetoelastic structure computed is

$$
\bar{p}_{\text {opt }}=0.375 \mu^{-1} \frac{\Lambda_{3}}{\Lambda_{4}}
$$

where

$\Lambda_{3}$

$$
=\left(\begin{array}{c}
1+\frac{5}{2} \chi^{2}\left(1-\frac{\mu_{0}}{\mu_{m}}\right)+\frac{91}{10} \chi^{4}\left(1-\frac{\mu_{0}}{\mu_{m}}\right)^{2}+\frac{143}{20} \chi^{6}\left(1-\frac{\mu_{0}}{\mu_{m}}\right)^{3} \\
-\frac{13}{10} \chi^{8}\left(1-\frac{\mu_{0}}{\mu_{m}}\right)^{4}+\frac{7}{200} \chi^{10}\left(1-\frac{\mu_{0}}{\mu_{m}}\right)^{5}+\frac{17}{12} \chi^{12}\left(1-\frac{\mu_{0}}{\mu_{m}}\right)^{6}
\end{array}\right),
$$

$\Lambda_{4}$

$$
=\left(\begin{array}{c}
1+\frac{15}{2} \chi^{2}\left(1-\frac{\mu_{0}}{\mu_{m}}\right)-\frac{683}{100} \chi^{4}\left(1-\frac{\mu_{0}}{\mu_{m}}\right)^{2}+\frac{107}{20} \chi^{6}\left(1-\frac{\mu_{0}}{\mu_{m}}\right)^{3} \\
-\frac{49}{50} \chi^{8}\left(1-\frac{\mu_{0}}{\mu_{m}}\right)^{4}+\frac{3}{100} \chi^{10}\left(1-\frac{\mu_{0}}{\mu_{m}}\right)^{5}+\frac{16}{25} \chi^{12}\left(1-\frac{\mu_{0}}{\mu_{m}}\right)^{6}
\end{array}\right),
$$

and for the special case $\chi \rightarrow 0$, the optimum clamping pressure reduce to the form $\widehat{\bar{p}}_{0}=0.375 \mu^{-1}=3 / 8 \mu$ in Damisa [25]. At this optimal pressure, the corresponding energy dissipation is

$$
\begin{aligned}
& \bar{D}_{\max }=\frac{3}{2} \Psi \\
& \times\left(\begin{array}{l}
(2+\varepsilon)-\Psi\left(1+\frac{421}{320} \varepsilon\right)-\Psi \frac{261}{640} \varepsilon^{2} \\
+\chi^{2}\left(1-\frac{\mu_{0}}{\mu_{m}}\right)\left(20-5 \varepsilon-\Psi\left(\frac{15}{2}+\frac{519}{80} \varepsilon+\frac{219}{160} \varepsilon^{2}\right)\right) \\
+\chi^{4}\left(1-\frac{\mu_{0}}{\mu_{m}}\right)^{2}\left(-\frac{91}{10}(2+\varepsilon)+\Psi\left(\frac{273}{40}+\frac{21}{16} \varepsilon-\frac{21}{20} \varepsilon^{2}\right)\right) \\
+\chi^{6}\left(1-\frac{\mu_{0}}{\mu_{m}}\right)^{3}\left(\frac{143}{20}(2+\varepsilon)-\Psi\left(\frac{429}{80}+\frac{327}{160} \varepsilon-\frac{153}{320} \varepsilon^{2}\right)\right) \\
+\chi^{8}\left(1-\frac{\mu_{0}}{\mu_{m}}\right)^{4}\left(-\frac{13}{10}(2+\varepsilon)-\Psi\left(\frac{39}{40}-\frac{13}{10} \varepsilon-\frac{33}{320} \varepsilon^{2}\right)\right) \\
+\chi^{10}\left(1-\frac{\mu_{0}}{\mu_{m}}\right)^{5}\left(\frac{7}{100}(1+5 \varepsilon)-\Psi\left(\frac{21}{800}+\frac{69}{1600} \varepsilon-\frac{9}{320} \varepsilon^{2}\right)\right) \\
+\chi^{12}\left(1-\frac{\mu_{0}}{\mu_{m}}\right)^{6}\left(\frac{17}{100}+\frac{2}{25} \varepsilon-\Psi\left(\frac{51}{800}+\frac{3}{100} \varepsilon^{2}\right)\right)
\end{array}\right)
\end{aligned}
$$

where

$\Psi=\frac{M_{1}}{M_{2}}$

$M_{1}$

$=\left(\begin{array}{c}1+\frac{5}{2} \chi^{2}\left(1-\frac{\mu_{0}}{\mu_{m}}\right)+\frac{91}{10} \chi^{4}\left(1-\frac{\mu_{0}}{\mu_{m}}\right)^{2}+\frac{143}{20} \chi^{6}\left(1-\frac{\mu_{0}}{\mu_{m}}\right)^{3} \\ -\frac{13}{10} \chi^{8}\left(1-\frac{\mu_{0}}{\mu_{m}}\right)^{4}+\frac{7}{200} \chi^{10}\left(1-\frac{\mu_{0}}{\mu_{m}}\right)^{5}+\frac{17}{12} \chi^{12}\left(1-\frac{\mu_{0}}{\mu_{m}}\right)^{6}\end{array}\right)$,

$M_{2}$

$=\left(\begin{array}{c}1+\frac{15}{2} \chi^{2}\left(1-\frac{\mu_{0}}{\mu_{m}}\right)-\frac{683}{100} \chi^{4}\left(1-\frac{\mu_{0}}{\mu_{m}}\right)^{2}+\frac{107}{20} \chi^{6}\left(1-\frac{\mu_{0}}{\mu_{m}}\right)^{3} \\ -\frac{49}{50} \chi^{8}\left(1-\frac{\mu_{0}}{\mu_{m}}\right)^{4}+\frac{3}{100} \chi^{10}\left(1-\frac{\mu_{0}}{\mu_{m}}\right)^{5}+\frac{16}{25} \chi^{12}\left(1-\frac{\mu_{0}}{\mu_{m}}\right)^{6}\end{array}\right) M$,

which indicates that even when linear pressure variation is admitted, the maximum dissipated energy still remains independent of the coefficient of friction as reported in [19]. 


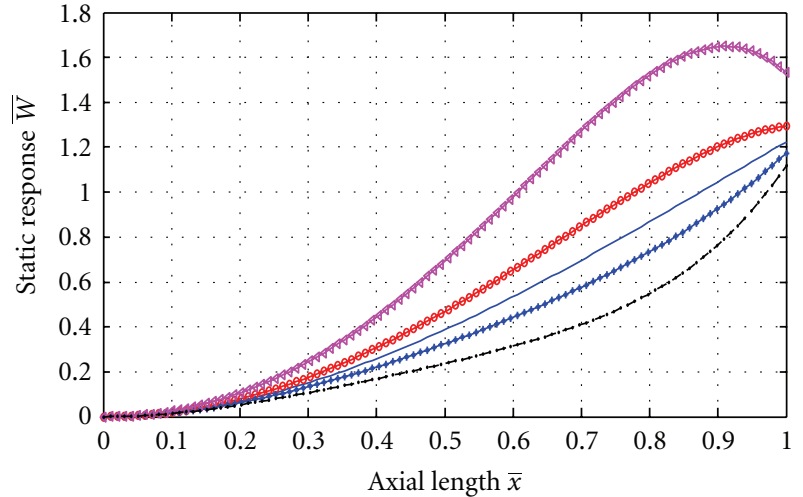

$$
\begin{aligned}
-\varepsilon & =0 & \multimap \varepsilon & =0.6 \\
\multimap \varepsilon & =-0.2 & -+\varepsilon & =0.6
\end{aligned}
$$

Figure $2: \bar{W}$ versus $\bar{x}$ with different values of $\varepsilon$ for the following case: $\chi=0.05 ; \mu_{r}=0.6 ; \mu \bar{P}_{0}=\mu \bar{P}_{\text {opt }}$.

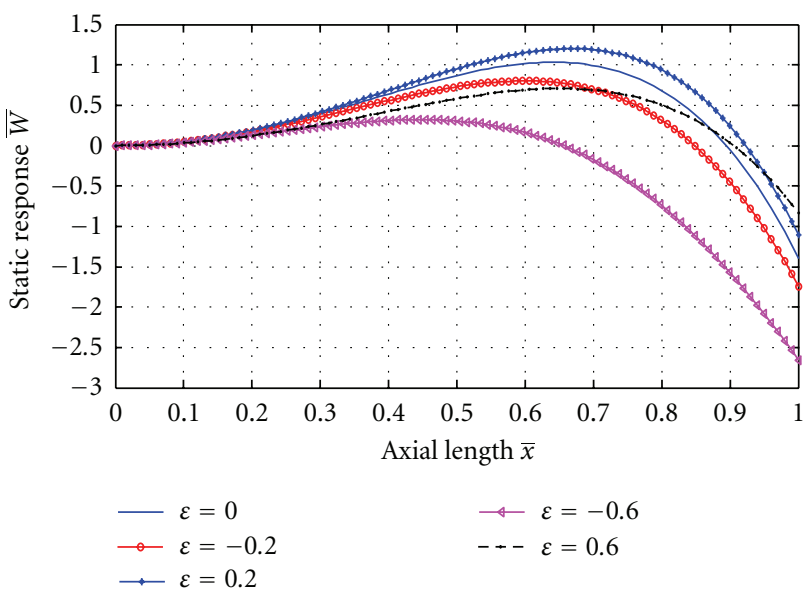

Figure 3: $\bar{W}$ versus $\bar{x}$ with different values of $\varepsilon$ for the following case: $\chi=0.5 ; \mu_{r}=0.6 ; \mu \bar{P}_{0}=\mu \bar{P}_{\text {opt }}$.

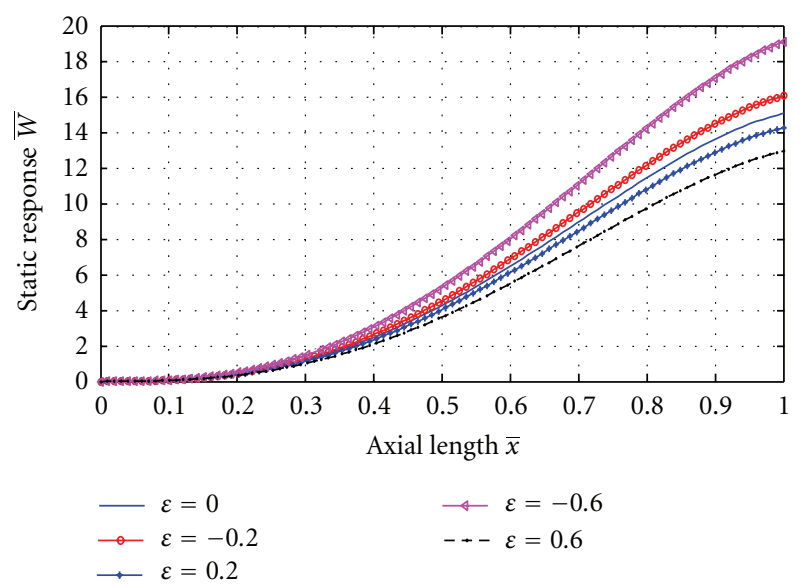

Figure 4: $\bar{W}$ versus $\bar{x}$ with different values of $\varepsilon$ for the following case: $\chi=1.5 ; \mu_{r}=0.6 ; \mu \bar{P}_{0}=\mu \bar{P}_{\text {opt }}$.
TABLE 1: Material and geometric parameters.

\begin{tabular}{lcc}
\hline Definition & Symbol & Value \\
\hline Magnetic permeability & $\mu_{0}$ & $4 \pi \times 10^{-7} \mathrm{mho} / \mathrm{m}$ \\
Interfacial pressure & $P$ & $1 \times 10^{9} \mathrm{Nm}^{-2}$ \\
Geometry & & \\
$\quad$ Length & $L$ & $0.1 \mathrm{~m}-5 \mathrm{~m}$ \\
$\quad$ Width & $b$ & $0.3 \mathrm{~m}$ \\
$\quad$ Thickness & $h$ & $0.001 \mathrm{~m}-0.4 \mathrm{~m}$ \\
Modulus of rigidity of materials & $E$ & $1.2 \times 10^{11} \mathrm{Nm}^{-2}$ \\
Density of material & $\rho$ & $2810 \mathrm{~kg} \mathrm{~m}^{-3}$ \\
\hline
\end{tabular}

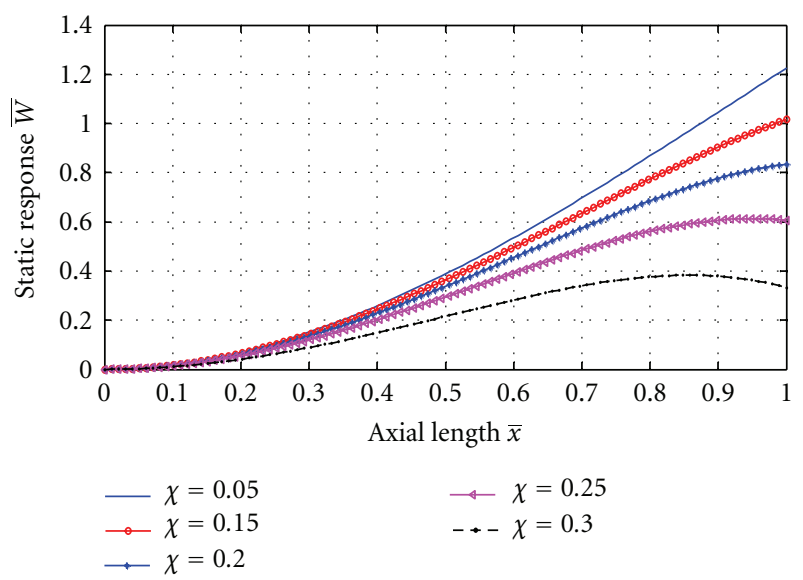

Figure 5: $\bar{W}$ versus $\bar{x}$ with different values of $\chi$ for the following case: $\varepsilon=0 ; \mu_{r}=0.6 ; \mu \bar{P}_{0}=\mu \bar{P}_{\text {opt }}$.

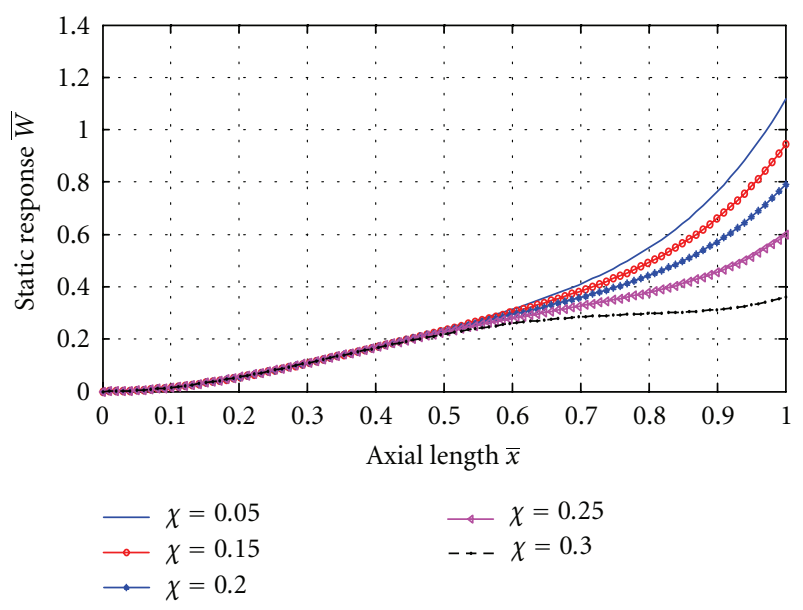

Figure 6: $\bar{W}$ versus $\bar{x}$ with different values of $\chi$ for the following case: $\mathcal{E}=0.6 ; \mu_{r}=0.6 ; \mu \bar{P}_{0}=\mu \bar{P}_{\text {opt }}$.

5.2. Analysis of Damping Capacity of the Magnetoelastic Structure. The damping capacity of any structure is a measure of the ratio of its slip energy dissipation to total strain energy under any conditions. For this case, we shall first derive the expression for the total strain energy following Damisa et al. [19]. It is a combination of the energy introduced by the bending moment as well as that stored from the deflection of the free end. While the former can be evaluated from 
the theorem of Castigliano, the later can be computed from where the free end deflection theory. For this problem, we can derive the total strain energy expression as

$$
\bar{X}=\bar{X}_{1}+\bar{X}_{2},
$$

$$
\begin{aligned}
& \bar{X}_{1}=\left(\begin{array}{l}
1-\mu \bar{p}_{0}(2+\varepsilon)+\mu^{2} \bar{p}_{0}{ }^{2}\left(1+\varepsilon+\frac{\varepsilon^{2}}{4}\right)+\chi^{2}\left(1-\frac{\mu_{0}}{\mu_{m}}\right)\left(1-\mu \bar{p}_{0}(2+\varepsilon) \bar{W}_{\max }\right) \\
+\chi^{4}\left(1-\frac{\mu_{0}}{\mu_{m}}\right)^{2} \bar{W}_{\max }{ }^{2}
\end{array}\right), \\
& \forall \bar{W}_{\max }=\left(\begin{array}{c}
1+\chi^{2}\left(1-\frac{\mu_{0}}{\mu_{m}}\right) \\
+\frac{3}{20} \chi^{4}\left(1-\frac{\mu_{0}}{\mu_{m}}\right)^{2}
\end{array}\right)\left(\begin{array}{l}
\left(1-\mu \bar{P}_{0}\left(1+\frac{\varepsilon}{2}\right)-\frac{\mu \bar{P}_{0} \varepsilon}{240}\right) \\
+\frac{4}{5} \chi^{2}\left(1-\frac{\mu_{0}}{\mu_{m}}\right)\left(1-\mu \bar{P}_{0}\left(1+\frac{\varepsilon}{2}\right)+\frac{\mu \bar{P}_{0} \varepsilon}{576}\right)
\end{array}\right),
\end{aligned}
$$

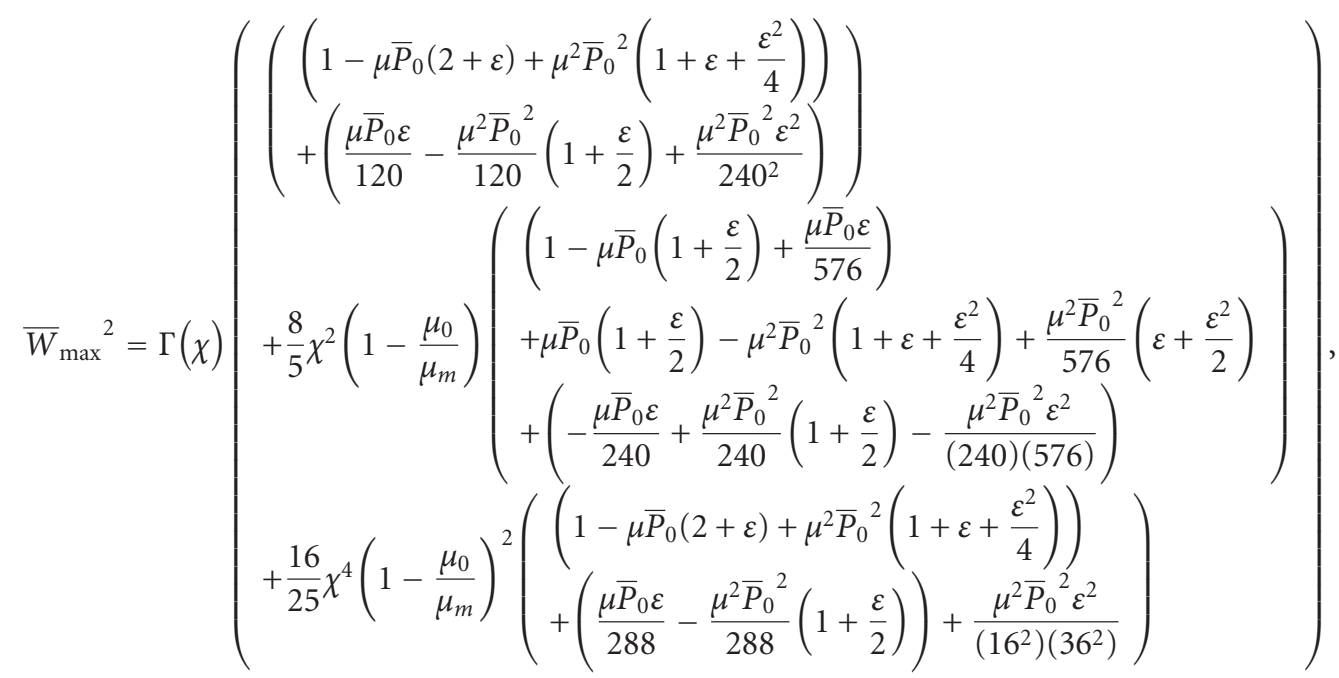

where

$$
\Gamma(\chi)=\left(1+\chi^{2}\left(1-\frac{\mu_{0}}{\mu_{m}}\right)+\frac{3}{20} \chi^{4}\left(1-\frac{\mu_{0}}{\mu_{m}}\right)^{2}\right) ;
$$

while

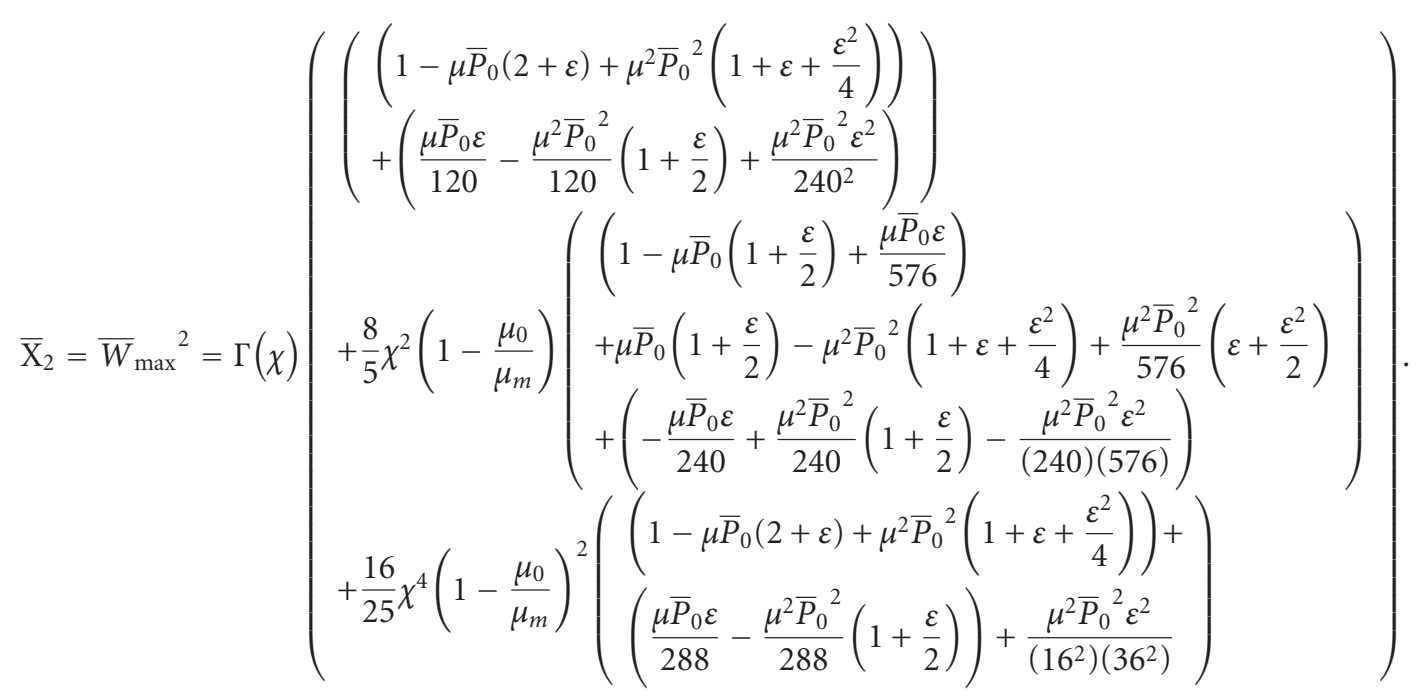


For this problem, the damping capacity following Damisa et al. [19] is

$$
\Phi=\frac{\bar{D}}{\bar{X}}
$$

whilst the maximum damping capacity can be computed as

$$
\Phi_{\max }=\frac{\bar{D}_{\max }}{\overline{\mathrm{X}}_{\max }}
$$

where

$$
\begin{aligned}
& \bar{X}_{\max }=\hat{\bar{X}}_{1}+\hat{\bar{X}}_{2} \text {, } \\
& \widehat{\bar{X}}_{1}=\left(\begin{array}{l}
1-\frac{3}{8} \Psi(2+\varepsilon)+\frac{9}{64} \Psi^{2}\left(1+\varepsilon+\frac{\varepsilon^{2}}{4}\right)+\chi^{2}\left(1-\frac{\mu_{0}}{\mu_{m}}\right)\left(1-\frac{3}{8} \Psi(2+\varepsilon) \widehat{W}_{\max }\right) \\
+\chi^{4}\left(1-\frac{\mu_{0}}{\mu_{m}}\right)^{2} \widehat{W}_{\max }^{2}
\end{array}\right), \\
& \forall \widehat{W}_{\max }=\left(\begin{array}{c}
1+\chi^{2}\left(1-\frac{\mu_{0}}{\mu_{m}}\right) \\
+\frac{3}{20} \chi^{4}\left(1-\frac{\mu_{0}}{\mu_{m}}\right)^{2}
\end{array}\right)\left(\begin{array}{c}
\left(1-\frac{3}{8} \Psi\left(1+\frac{\varepsilon}{2}\right)-\frac{\Psi \varepsilon}{640}\right) \\
+\frac{4}{5} \chi^{2}\left(1-\frac{\mu_{0}}{\mu_{m}}\right)\left(1-\frac{3}{8} \Psi\left(1+\frac{\varepsilon}{2}\right)+\frac{\Psi \varepsilon}{(8)(192)}\right)
\end{array}\right) \\
& \widehat{W}_{\text {max }}^{2}=\Gamma(\chi)\left(\begin{array}{c}
\left(\begin{array}{c}
\left(1-\frac{3}{8} \Psi(2+\varepsilon)+\frac{9}{64} \Psi^{2}\left(1+\varepsilon+\frac{\varepsilon^{2}}{4}\right)\right) \\
+\left(\frac{\Psi \varepsilon}{320}-\frac{3 \Psi^{2}}{(40)(64)}\left(1+\frac{\varepsilon}{2}\right)+\frac{\Psi^{2} \varepsilon^{2}}{\left(10^{2}\right)\left(8^{4}\right)}\right)
\end{array}\right) \\
+\frac{8}{5} \chi^{2}\left(1-\frac{\mu_{0}}{\mu_{m}}\right)\left(\begin{array}{l}
\left(1-\frac{3}{8} \Psi\left(1+\frac{\varepsilon}{2}\right)+\frac{\Psi \varepsilon}{(8)(192)}\right) \\
+\frac{3}{8} \Psi\left(1+\frac{\varepsilon}{2}\right)-\frac{9}{64} \Psi^{2}\left(1+\varepsilon+\frac{\varepsilon^{2}}{4}\right)+\frac{\Psi^{2}}{64^{2}}\left(\varepsilon+\frac{\varepsilon^{2}}{2}\right) \\
+\left(-\frac{\Psi \varepsilon}{640}+\frac{\Psi^{2}}{\left(10^{2}\right)\left(8^{4}\right)}\left(1+\frac{\varepsilon}{2}\right)-\frac{\Psi^{2} \varepsilon^{2}}{\left(64^{2}\right)(240)}\right)
\end{array}\right) \\
+\frac{16}{25} \chi^{4}\left(1-\frac{\mu_{0}}{\mu_{m}}\right)
\end{array}\right),
\end{aligned}
$$

while

$$
\widehat{\bar{X}}_{2}=\widehat{W}_{\max }^{2} \text {. }
$$

\section{Analysis of Results}

In this paper, static slip and energy dissipation in twolayer sandwich homogeneous elastic beams in a transverse magnetic field is studied. The problem physics derives from energy dissipation via contact frictional stresses in press fit joints. In the contrived problem, a constant tip force prompts the two-layer cantilever elastic beams in an environment of uniform transverse magnetic field. Simulation studies employed the characteristic values listed in Table 1. (Figures $2,3,4,5,6,7,8,9,10,11,12,13$, and 14) illustrate the effect of magnetoelasticity, relative permeability, and pressure gradient at optimum interface clamping pressure. In Figures 2-7, a tip force in an environment of weak magnetic field $(\chi=0.05)$ prompts the structure. We displayed in the Figure 2 result for homogeneous laminates of relative permeability $\mu_{r}=0.6$.
As can be seen, the deflection in the absence of pressure gradient $(\varepsilon=0)$ is lower and higher than the cases of negative and positive pressure gradients. Such a response pattern is expected ideally.

The rigidity or stiffness of the structure from the fixed end should increase with progressive increment in the tightening torque axially. It is conceivable to expect higher deflection for the case $\varepsilon=-0.6$ than for $\varepsilon=-0.2$. Intuitively, a converse pattern should be expected in the reversed order as correctly noted in the figure. Furthermore, for $\varepsilon=$ -0.6 , there is a tendency for buckling to occur with axial progressive decrement in the tightening torque close to the free end, whilst for $\varepsilon=-0.2$, buckling is expected to occur at the free end. In Figure 3, the magnetic field $(\chi=0.5)$ intensity is relatively higher. Under the same parametric variables, we note a reversion in the pattern of deflection and tendency for buckling in the neighborhood of the free end irrespective of the value of $\varepsilon$.

The effect of much higher magnetic field intensity ( $\chi=$ 1.5) on the deflection response is illustrated in Figure 4. 


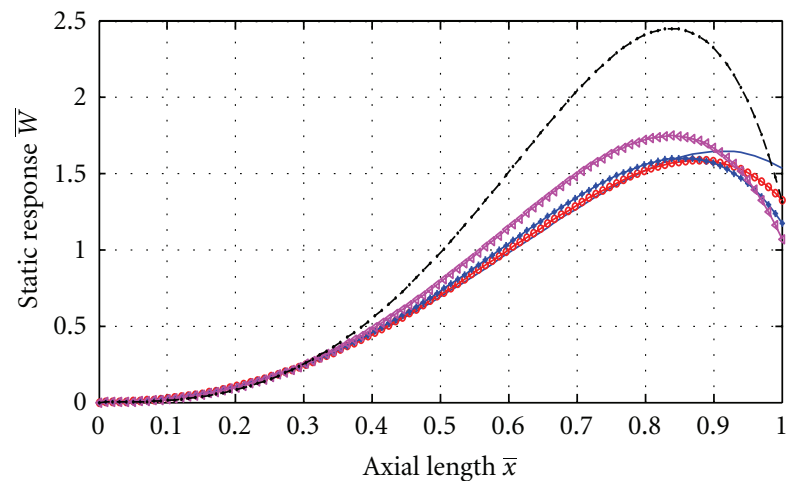

$$
\begin{array}{rlrl}
-\chi & =0.05 & \hookrightarrow \chi & =0.25 \\
\multimap \chi & =0.15 & -\multimap \chi & =0.3 \\
\rightarrow \chi & =0.2 &
\end{array}
$$

Figure 7: $\bar{W}$ versus $\bar{x}$ with different values of $\chi$ for the following case: $\varepsilon=-0.6 ; \mu_{r}=0.6 ; \mu \bar{P}_{0}=\mu \bar{P}_{\text {opt }}$.

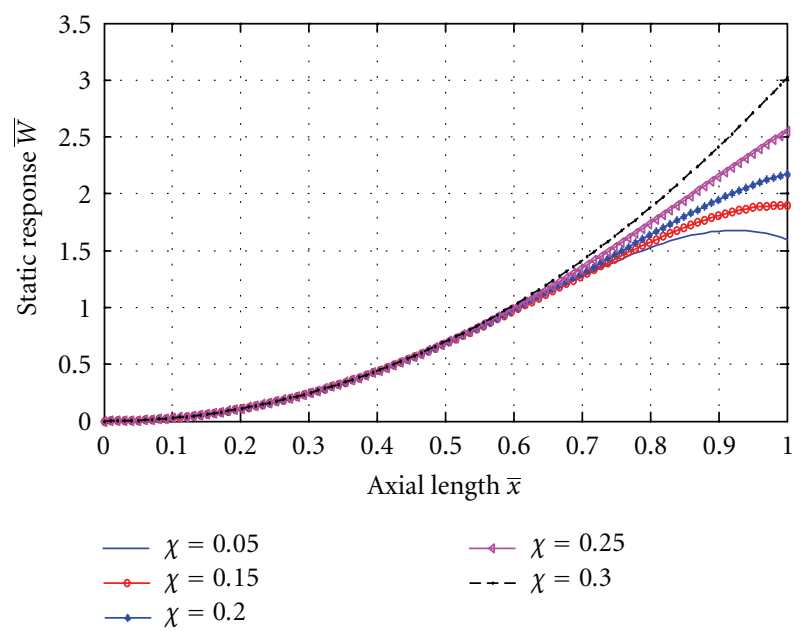

Figure 8: $\bar{W}$ versus $\bar{x}$ with different values of $\chi$ for the following case: $\varepsilon=-0.6 ; \mu_{r}=6 ; \mu \bar{P}_{0}=\mu \bar{P}_{\text {opt }}$.

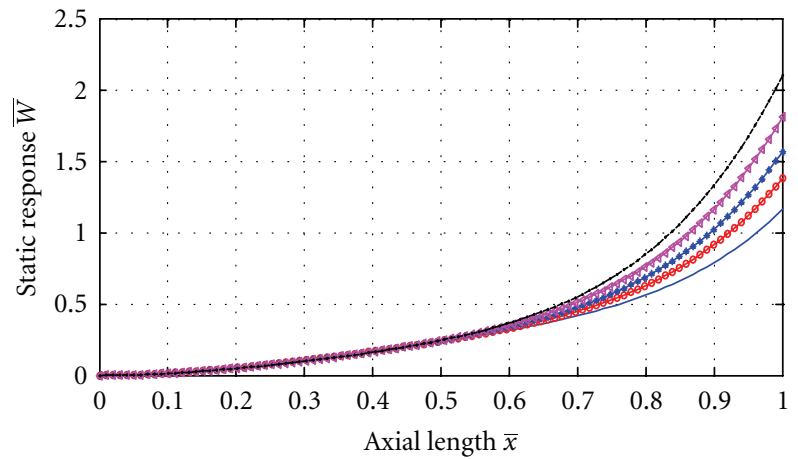

$$
\begin{array}{rlrl}
-\chi & =0.05 & \because \chi & =0.25 \\
\because \chi & =0.15 & --\chi=0.3 \\
\rightarrow \chi & =0.2 &
\end{array}
$$

FIgURE 9: $\bar{W}$ versus $\bar{x}$ with different values of $\chi$ for the following case: $\varepsilon=0.6 ; \mu_{r}=6 ; \mu \bar{P}_{0}=\mu \bar{P}_{\text {opt }}$.

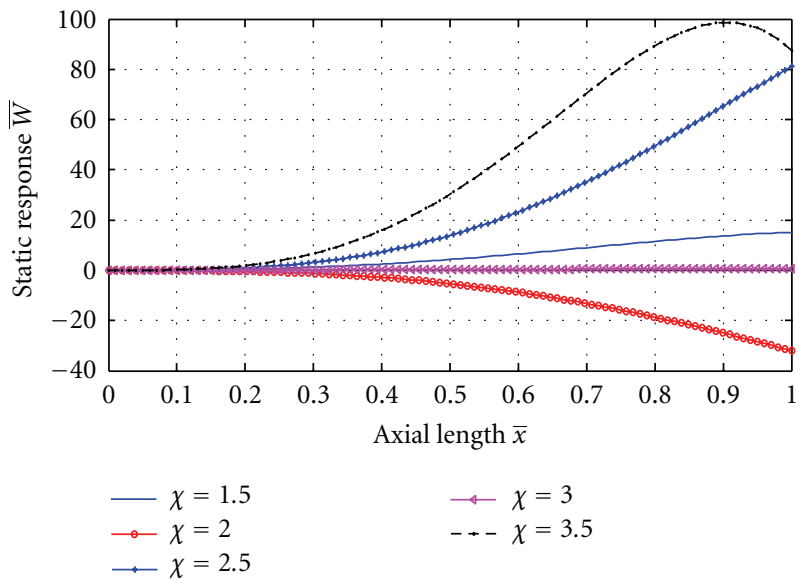

FIgURE 10: $\bar{W}$ versus $\bar{x}$ with different values of $\chi$ for the following case: $\varepsilon=0 ; \mu_{r}=6 ; \mu \bar{P}_{0}=\mu \bar{P}_{\text {opt }}$.

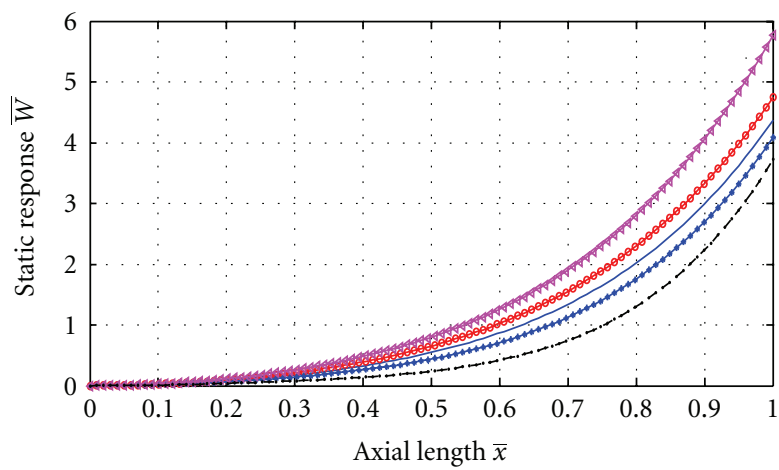

$$
\begin{aligned}
-\varepsilon & =0 \\
\multimap \varepsilon & =-0.2 \\
\multimap \varepsilon & --\varepsilon=0.6
\end{aligned}
$$$$
\longrightarrow \varepsilon=0.2
$$

Figure 11: $\bar{W}$ versus $\bar{x}$ with different values of $\varepsilon$ for the following case: $\chi=0.5 ; \mu_{r}=6 ; \mu \bar{P}_{0}=\mu \bar{P}_{\text {opt }}$.

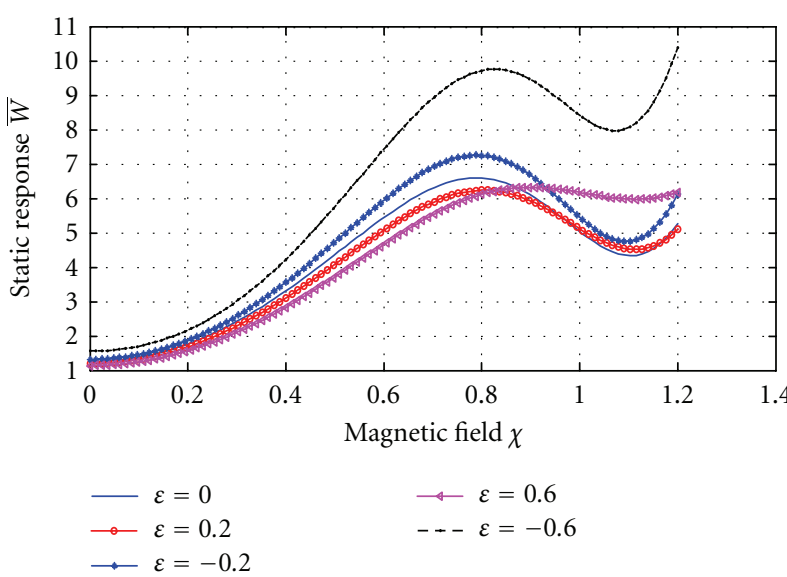

Figure 12: $\bar{W}$ versus $\chi$ with different values of $\varepsilon$ for the following case: $\bar{x}=1 ; \mu_{r}=6 ; \mu \bar{P}_{0}=\mu \bar{P}_{\text {opt }}$. 
Nature of curves indicated identical trajectories with higher deflection and symmetric ordering with respect to the role of the pressure gradient. In Figure 5, we illustrate the effect of varying the magnetic field intensity for the case of uniform clamping pressure on the static deflection profile. In the environment of lower magnetic field intensity $\chi$, the trajectory is uniform but as field intensity increases, proportional decrements to respective optimum points are noted. Next, we display in Figures 6 and 7 the effects of pressure gradients on the deflection pattern in the same magnetic field environment. For the case $\varepsilon=0.6$, the pattern is the same from the fixed end to the middle of the structure. Beyond this point, the effect of the field intensity becomes noticeable with higher deflection in the weak magnetic environment. We also note identical pattern from the fixed end to the middle of the structure for the case $\varepsilon=-0.6$. Beyond this point, the effect of the field intensity becomes noticeable. Natures of curves indicate higher deflection with respective optimum points in the strong magnetic environment. Next, we show in Figures 8 and 9 the effect of increasing the relative permeability on the static deflection.

As shown in Figures 8 and 9, the effect of increasing the field intensity is noticeable beyond the middle of the structure. Nevertheless, for the case $\varepsilon=-0.6$, a bisegmented asymmetrical deflection appeared in the neighborhood of the free end of the structure compared to the case $\varepsilon=0.6$ where the pattern of deflection is progressively monotonic. Profiles for the case $\varepsilon=0$ (uniform pressure) in much higher field intensity are illustrated in Figure 10. In contrast to results in Figures 8 and 9, we note a very visible bisegmented asymmetrical deflection slightly away from the fixed point to the free end of the structure. Next, we study the static response of the structure at optimum clamping pressure for the case displayed in Figure 11. Nature of curves indicate that the deflections for negative pressure gradient are higher than for the case of uniform pressure and positive gradient as reported in [19].

The cumulative effect of pressure gradient and field intensity on the mid structure and tip deflections are demonstrated in Figures 12 and 13. The picture in Figure 12 indicates a three-region segmentation except for the case $\varepsilon=0.6$ as modulated by the field intensity. In the first region, the field intensity lies within the range $0<\chi \leq 0.2$. Here, the deflections are ordered in consonant with the forms and values of pressure gradient $\varepsilon$ with each profile, indicating a monotonic variable that is approaching a local maximum.

In the second region, the field intensity lies within the range $0.5 \leq \chi \leq 1$. Here, each profile is also a monotonic variable approaching a local minimum, and in the last region, the field intensity lies within the range $1 \leq \chi \leq 1.2$ and the respective tip deflection is progressively monotonic. Next, the deflection pattern in the middle of the structure is displayed in Figure 13. In contrast to tip deflection, a relatively stable structure is noted in the range $0 \leq \chi \leq$ 0.4 and beyond, and a segmented distortional response characterized the structure. To what extent is the optimum clamping pressure influenced by the pressure gradient and

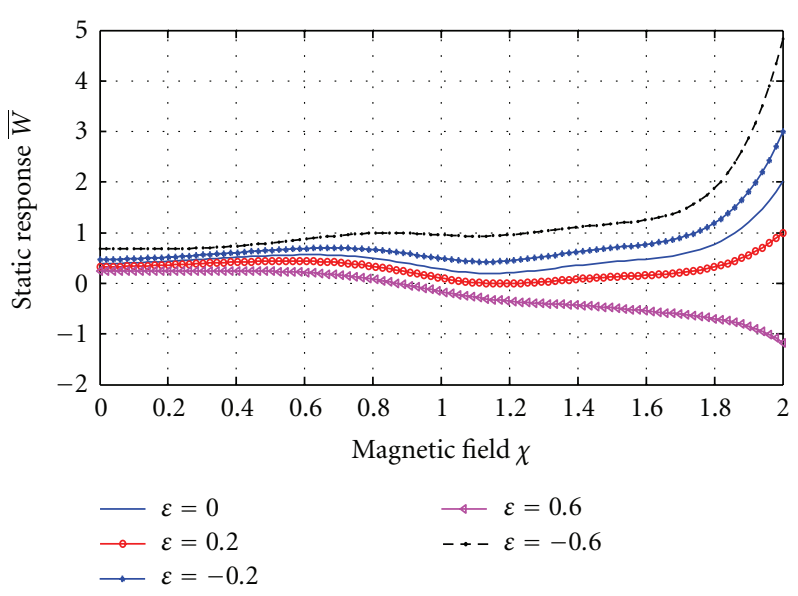

FIGURE 13: $\bar{W}$ versus $\chi$ with different values of $\varepsilon$ for the following case: $\bar{x}=0.5 ; \mu_{r}=6 ; \mu \bar{P}_{0}=\mu \bar{P}_{\text {opt }}$.

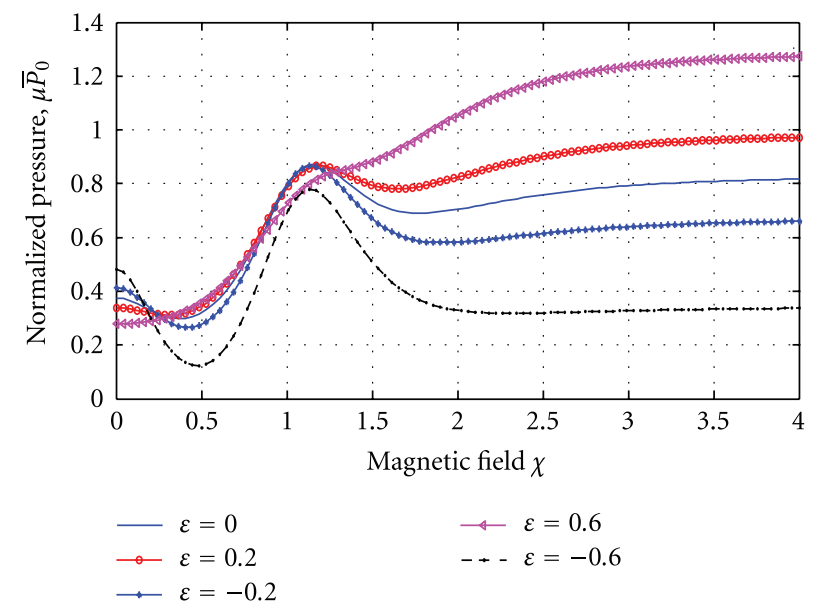

FIGURE 14: $\mu \bar{P}_{0}$ versus $\chi$ with different values of $\varepsilon$ for the following case: $\mu_{r}=6$.

the magnetic field strength? Such modulating effect is demonstrated in Figure 14.

In the range $0 \leq \chi \leq 1.5$, a cyclic propagation ensued and beyond, and the interface pressure becomes stable and higher for positive pressure gradient. The profiles of interfacial static slip are shown in Figures 15-21. Effect of pressure gradient on the tip static slip as a function of the magnetic field intensity is displayed in Figure 15. Family of curves indicate a three-region profile. In the first region, the slip for positive pressure gradient is higher than the cases for uniform pressure and negative pressure gradient. As the magnetic intensity increases to $\chi=0.2$, the interfacial slip becomes constant irrespective of the nature of the pressure gradient. Beyond this point, the second region begins and a reversal in the pressure gradient becomes apparent.

In this domain, each curve indicates a monotonic progressing slip that attains a maximum value subject to common critical magnetic field intensity. Next, we display in Figures 16, 17, and 18 gross interfacial slip profiles for 


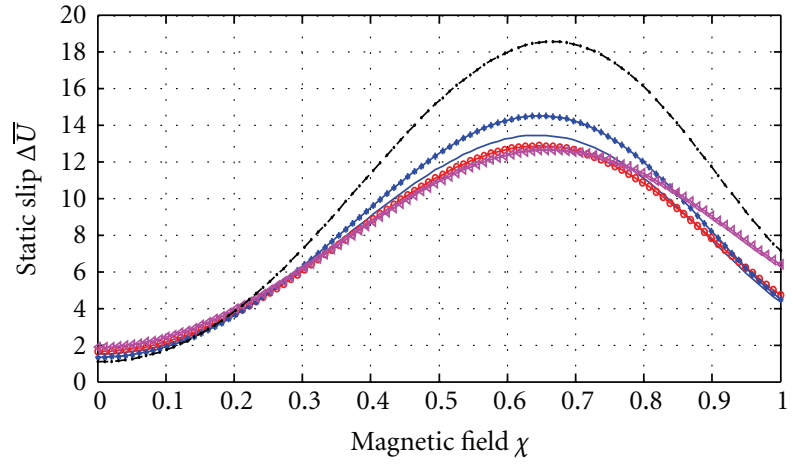

$$
\begin{aligned}
-\varepsilon & =0 & \leftarrow \varepsilon=0.6 \\
\multimap \varepsilon & =0.2 & -\varepsilon \varepsilon=-0.6 \\
\multimap \varepsilon & =-0.2 &
\end{aligned}
$$

FIgURE 15: $\Delta \bar{U}$ versus $\chi$ with different values of $\varepsilon$ for the following case: $\bar{x}=1 ; \mu_{r}=6$.

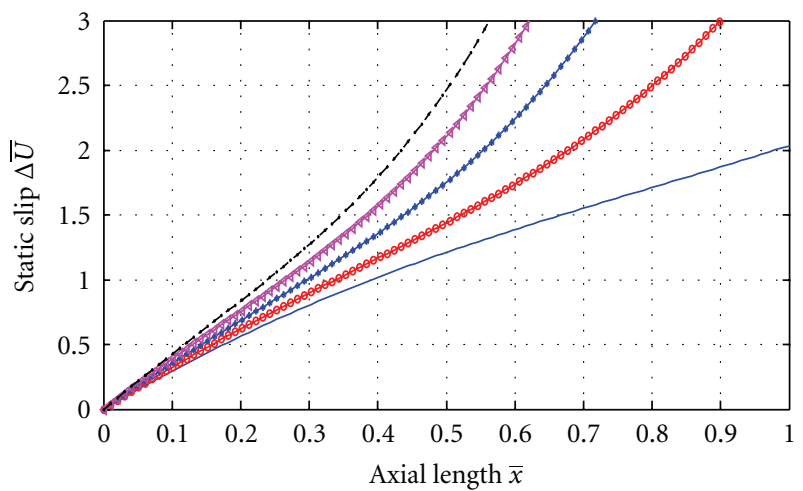

$$
\begin{aligned}
-\chi & =0 & \hookrightarrow \chi=0.3 \\
\rightarrow \chi & =0.1 & -\succ \chi=0.4 \\
\multimap \chi & =0.2 &
\end{aligned}
$$

Figure 16: $\Delta \bar{U}$ versus $\bar{x}$ with different values of $\chi$ for the following case; $\varepsilon=0 ; \mu_{r}=6 ; \mu \bar{P}_{0}=\mu \bar{P}_{\text {opt }}$.

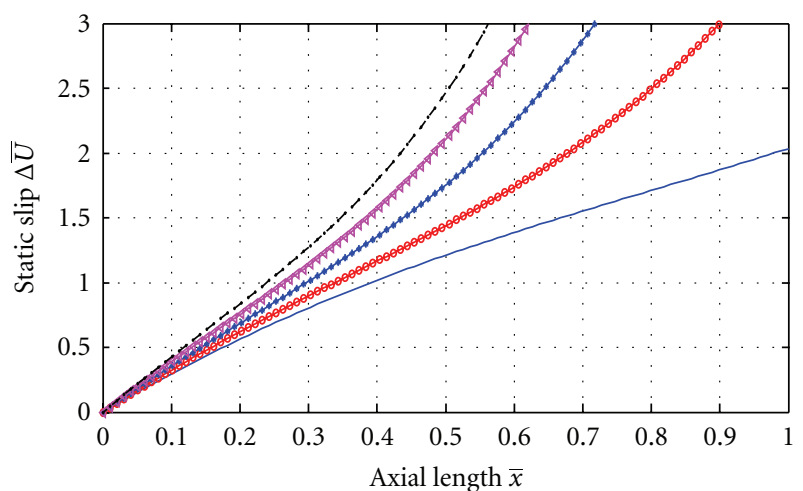

$$
\begin{array}{rlrl}
-\chi & =0 & \rightarrow & -40.3 \\
\rightarrow \chi & =0.1 & -\cdots \chi & =0.4 \\
\rightarrow \chi & =0.2 &
\end{array}
$$

Figure 17: $\Delta \bar{U}$ versus $\bar{x}$ with different values of $\chi$ for the following case: $\varepsilon=0.6 ; \mu_{r}=6 ; \mu \bar{P}_{0}=\mu \bar{P}_{\text {opt }}$.

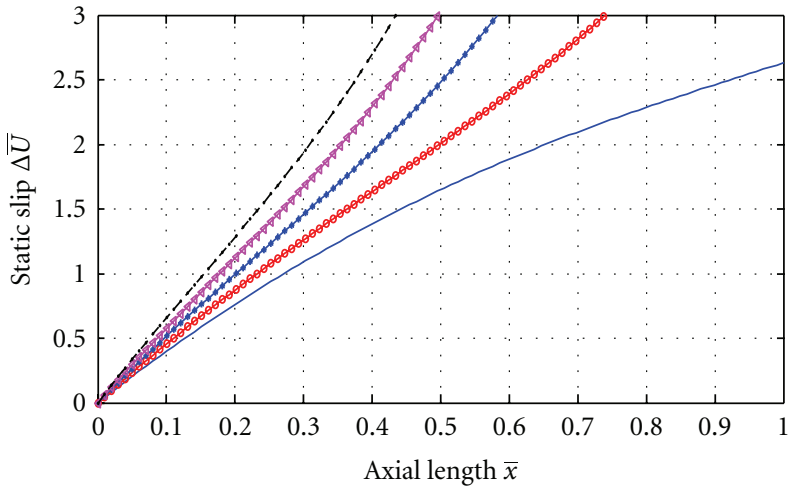

$$
\begin{array}{rlrl}
\longrightarrow \chi & =0 & \hookrightarrow \chi & =0.3 \\
\rightarrow \chi & =0.1 & --\chi=0.4 \\
\multimap \chi & =0.2 &
\end{array}
$$

FIGURE 18: $\Delta \bar{U}$ versus $\bar{x}$ with different values of $\chi$ for the following case: $\varepsilon=-0.6 ; \mu_{r}=6 ; \mu \bar{P}_{0}=\mu \bar{P}_{\text {opt }}$.

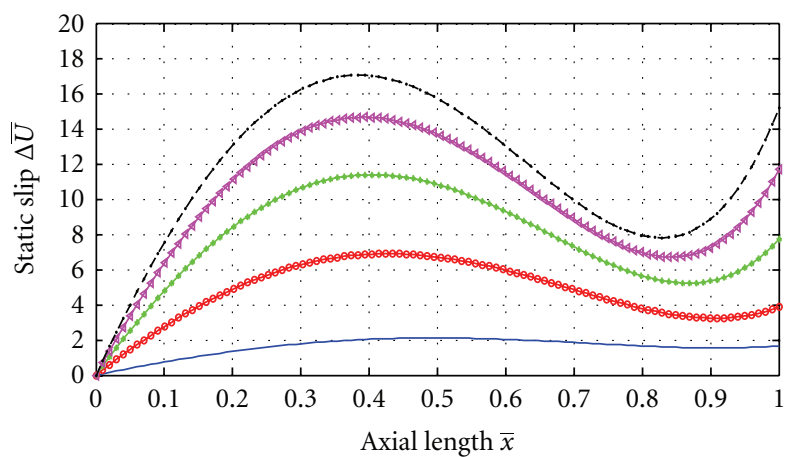

$$
\begin{aligned}
& -\mu_{r}=2 \quad-\leftarrow \mu_{r}=5 \\
& \because \mu_{r}=3 \quad--\mu_{r}=6 \\
& \longrightarrow \mu_{r}=4
\end{aligned}
$$

FIgURE 19: $\Delta \bar{U}$ versus $\bar{x}$ with different values of $\mu_{r}$ for the following case: $\varepsilon=0 ; \chi=1.5 ; \mu \bar{P}_{0}=\mu \bar{P}_{\text {opt }}$.

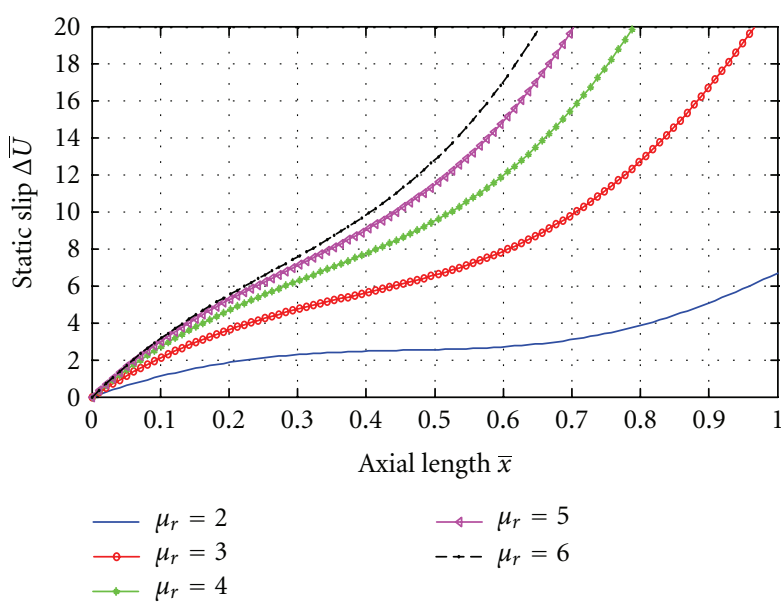

Figure 20: $\Delta \bar{U}$ versus $\bar{x}$ with different values of $\mu_{r}$ for the following case: $\varepsilon=0.6 ; \chi=1.5 ; \mu \bar{P}_{0}=\mu \bar{P}_{\text {opt }}$. 


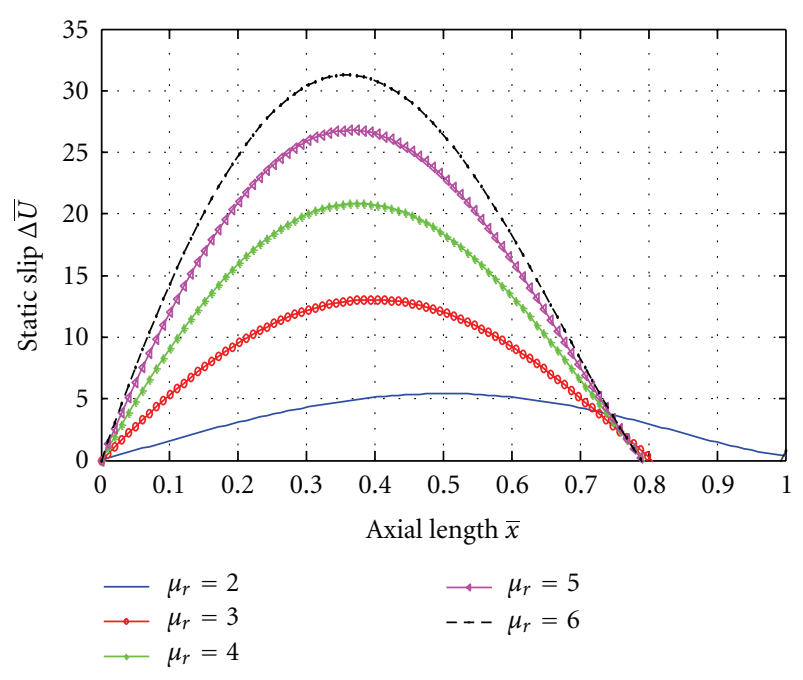

Figure 21: $\Delta \bar{U}$ versus $\bar{x}$ with different values of $\mu_{r}$ for the following case: $\varepsilon=-0.6 ; \chi=1.5 ; \mu \bar{P}_{0}=\mu \bar{P}_{\text {opt }}$.

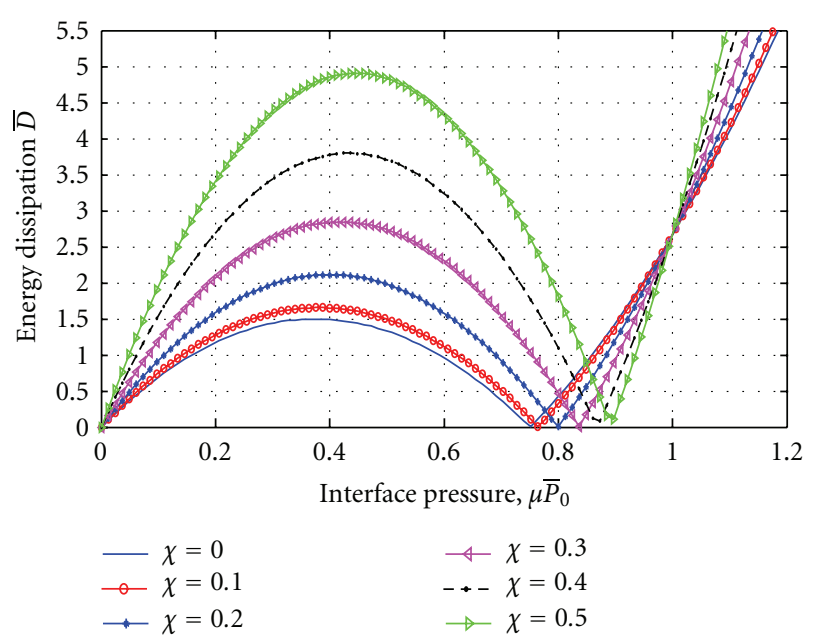

Figure 22: $\bar{D}$ versus $\mu \bar{P}_{0}$ with different values of $\chi$ for the following case: $\varepsilon=0 ; \mu_{r}=6$.

the cases of uniform pressure, positive- and negativepressure gradients, respectively. As noted in [19], gross slip for negative pressure gradients is higher compared to the cases of uniform pressure and positive pressure gradients.

For the special case, $\chi=0$, the profiles replicated the results in [19] for the same values of $\varepsilon$. Irrespective of the form of $\varepsilon$, the gross slip is proportional to the magnetic intensity and admits approximate linear profile when compared to the case $\chi=0$. The gross slip for uniform optimum pressure as influenced by different relative permeability values in an environment of strong magnetic field defined by $\chi=1.5$ is shown in Figure 19. In general, interfacial slip increases as the permeability increases. Apart from the curve typified by $\mu_{r}=2$, which rises progressively to an optimum value, the rest profiles admit cyclic variation with local maxima and minima values.

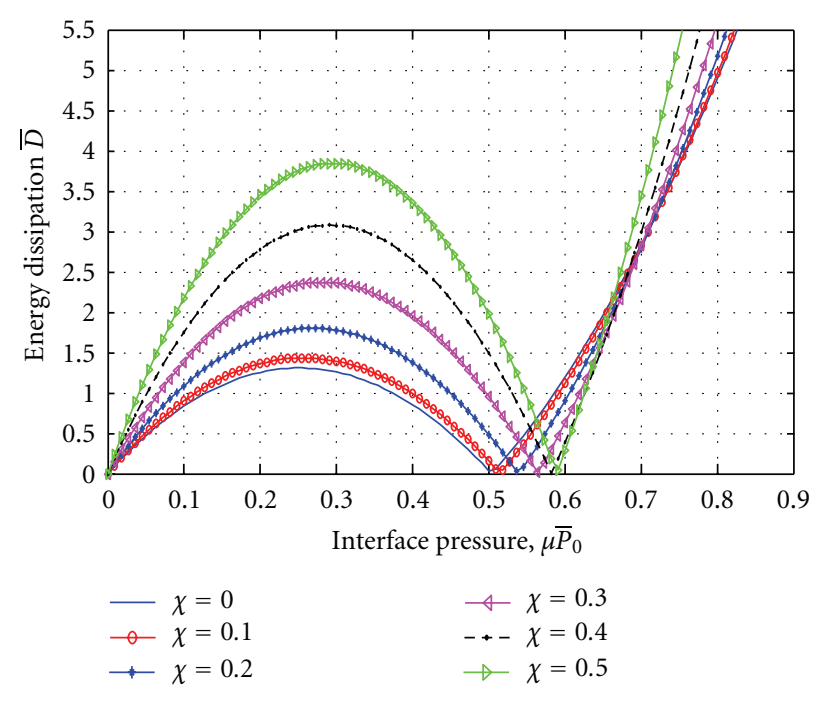

Figure 23: $\bar{D}$ versus $\mu \bar{P}_{0}$ with different values of $\chi$ for the following case: $\varepsilon=0.6 ; \mu_{r}=6$.

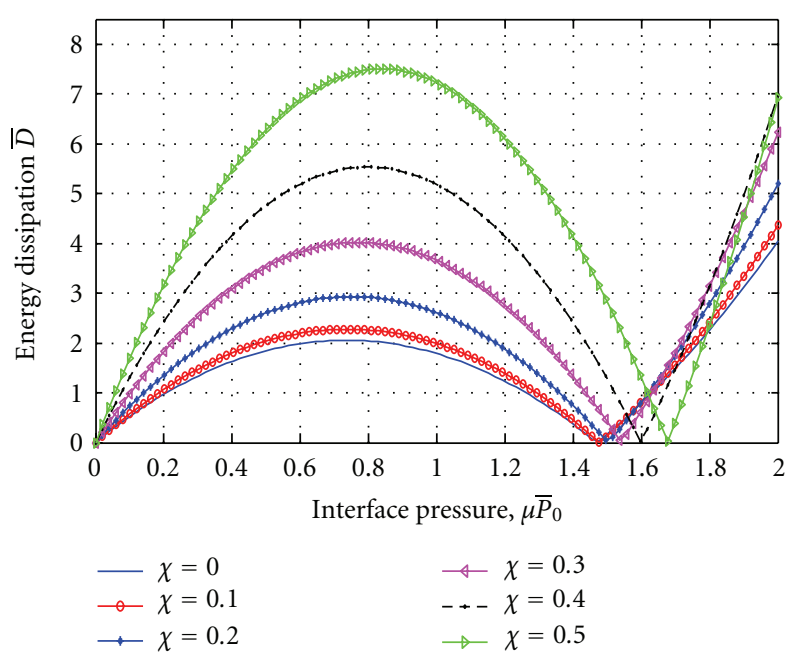

Figure 24: $\bar{X}$ versus $\mu \bar{P}_{0}$ with different values of $\chi$ for the following case: $\varepsilon=0.6 ; \mu_{r}=6$.

To what extent are the profiles modified for the cases of positive and negative pressure gradients? Such modification or effects are displayed in Figures 20 and 21 respectively. In Figure 20, profiles admit the same noncyclic configuration pattern that are proportional to the permeability values across the structures, and in Figure 21 slip profiles are proportionally parabolic curves. Energy dissipation ability as influenced by the magnetic field intensity and interfacial pressure are shown in Figures 22-24. Natures of profiles indicate a three-region regime. As shown in the plotted curves, the profiles are parabolic in the first region with local maxima as reported in $[19,20]$ and here, the interfacial pressure values are restricted in consonance with the form of pressure gradient. The same observations are noted in Damisa [25], where energy dissipation is maximum at respective optimum clamping pressure defined 


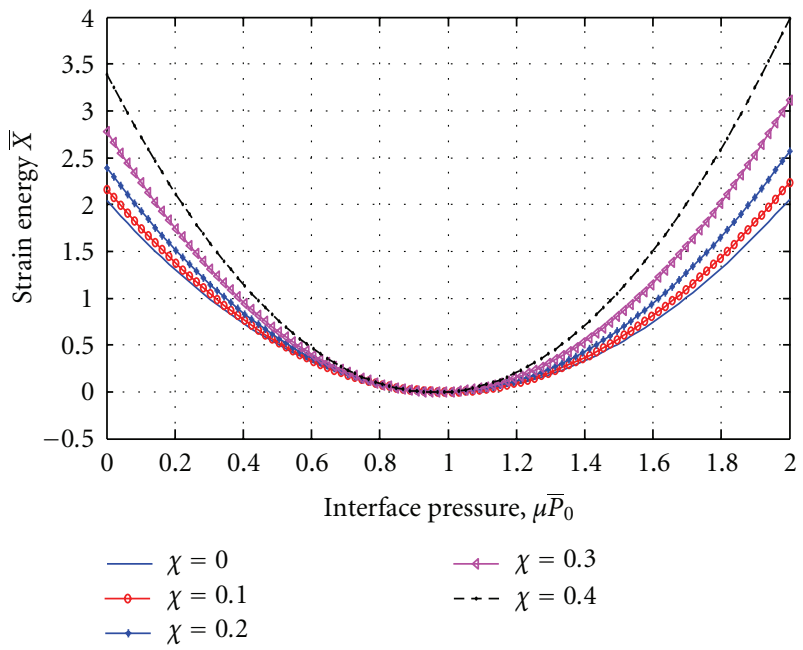

Figure 25: $\bar{X}$ versus $\mu \bar{P}_{0}$ with different values of $\chi$ for the following case: $\varepsilon=0 ; \mu_{r}=6$.

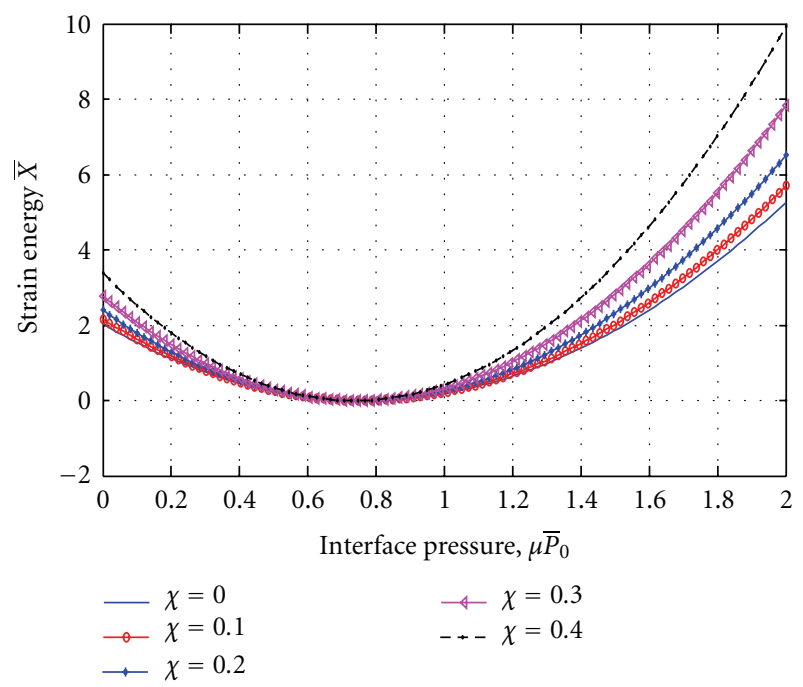

Figure 26: $\bar{D}$ versus $\mu \bar{P}_{0}$ with different values of $\chi$ for the following case: $\varepsilon=0.6 ; \mu_{r}=6$.

by corresponding critical transition into the second region. Such is anticipated via (72). In the second region, the values of the energy dissipation quanta are reversed in accordance with the respective magnetic field intensity prior to entering the third region. In this region, the dissipation quanta are proportional to the magnetic field intensities. The same trends are noted for energy dissipation profiles for the cases of positive and negative pressure gradients as shown in Figures 23 and 24.

As expected, the magnetic environment has an increasing effect on the energy dissipation mechanism as shown in Figures 22-24. Nevertheless, as correctly noted in $[19,20]$, dissipation is more with negative pressure gradient than for the uniform pressure and positive pressure gradient. The strain energy profiles as influenced by the magnetic field

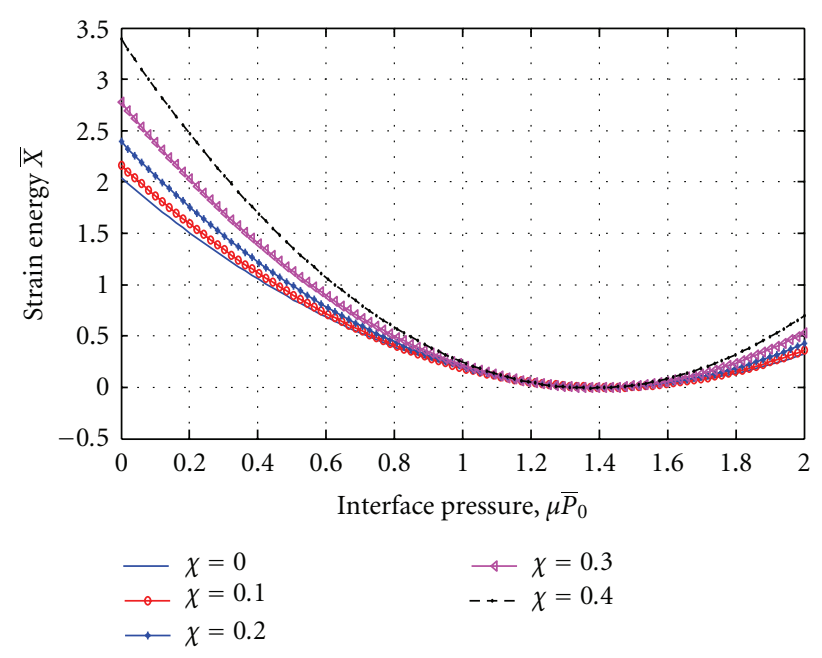

Figure $27: \bar{X}$ versus $\mu \bar{P}_{0}$ with different values of $\chi$ for the following case: $\varepsilon=-0.6 ; \mu_{r}=6$.

intensity and pressure gradient parameter are illustrated in Figures 25, 26, and 27. For the case of uniform pressure, symmetric parabolic curves proportional to the magnetic intensity ensued as shown in Figure 25, whilst skewed asymmetric parabolas are displayed for the positive and negative pressure gradients.

\section{Conclusion}

A well-posed mathematical physics problem on the mechanics of interfacial slip and energy dissipations mechanism with a two-layer sandwich homogenous elastic beam in a transverse magnetic field is presented. By employing operational methods, closed form polynomial expressions are derived for the responses defined in the body of the paper. In particular, the effects of magnetoelasticity and interfacial pressure gradient are demonstrated for design analysis and engineering applications. For special and limit cases, recent theoretical and experimental results are validated from the theory reported in the paper.

\section{Nomenclature}

$b$ : $\quad$ Width of laminated beam

$B_{0}$ : Magnetic flux density

$d / d x$ : Differential operator

E: $\quad$ Electric field intensity

E: Modulus of rigidity

F: $\quad$ Applied end force amplitude

$h$ : Depth of laminated beam

$H$ : $\quad$ Magnetic field intensity

I: $\quad$ Moment of inertia

$L: \quad$ Length of laminated beam

$P$ : Clamping pressure at the interface of the

laminated beams

$t: \quad$ Time coordinate

$u, v$ : Velocity 
$\dot{u}, \dot{v}:$ Acceleration

$U_{1}$ : Displacement of the lower laminate

$U_{2}$ : Displacement of the upper laminate

$W: \quad$ Dynamic response

$W^{F}$ : Dynamic response in Laplace transform plane

$\widetilde{W}: \quad$ Dynamic response in Fourier transform plane

$\widetilde{W}^{F}$ : Dynamic response in Fourier-Laplace transform plane

$\mathrm{W}^{\mathrm{F}}$ : Transverse response in Fourier plane

$x$ : $\quad$ space coordinate along the beam interface

$z$ : $\quad$ Space coordinate perpendicular to the beam interface

$\otimes: \quad$ Tensor Product

$\varepsilon$ : $\quad$ Pressure gradient

$\mu: \quad$ Dry friction coefficient

$\mu_{m}$ : Permeability of the medium

$\mu_{r}: \quad$ Relative permeability of the medium

$\chi$ : $\quad$ Normalized magnetic field intensity

$\varepsilon_{x 1}: \quad$ Axial strain in layer-1

$\varepsilon_{x 2}: \quad$ Axial strain in layer-2

$\gamma_{x z}:$ Angular strain

$\vec{h}(x)$ : Disturbed magnetic field vector

$\vec{H}(x)$ : Undisturbed magnetic field vector

$\vec{e}(x)$ : Disturbed electric field vector

$\vec{b}_{m}$ : Disturbed magnetic induction vector

$\tau^{M}: \quad$ Maxwell stress tensor

$I_{s}$ : Identity stress tensor.

\section{References}

[1] J. S. Lee, "Destabilizing effects of magnetic dampimg in plate strip," Journal of Engineering Mechanics, vol. 118, no. 1, pp. 161-173, 1992.

[2] F. C. Moon and Y. H. Pao, "Magnetoelastic buckling of a thin plate," Journal of Applied Mechanics, vol. 35, pp. 53-58, 1968.

[3] F. C. Moon and Y. H. Pao, "Vibration and dynamic instability of a beam-plate in a transverse magnetic field," Journal of Applied Mechanics, vol. 36, pp. 92-100, 1969.

[4] D. V. Wallerstein and M. O. Peach, "Magnetoelastic buckling of beams and thin plates of magnetically soft material," Journal of Applied Mechanics, vol. 39, pp. 451-455, 1972.

[5] K. Miya, T. Takagi, and Y. Ando, "Finite element analysis of magnetoelastic buckling of a ferromagnectic beam-plate," Journal of Applied Mechanics, vol. 47, no. 2, pp. 377-382, 1980.

[6] M. O. Peach, N. S. Christopherson, J. M. Dalrymple, and G. L. Viegelahn, "Magnetoelastic buckling: why theory and experiment disagree," Experimental Mechanics, vol. 28, no. 1, pp. 6569, 1988.

[7] J. S. Lee, "Dynamic stability of conducting beam-plates in transverse magnetic fields," Journal of Engineering Mechanics, vol. 122, no. 2, pp. 89-94, 1996.

[8] Y. H. Zhou, X. J. Zheng, and K. Miya, "Magnetoelastic bending and snapping of ferromagnetic plates in oblique magnetic fields," Fusion Engineering and Design, vol. 30, no. 4, pp. 325337, 1995.

[9] Y. H. Zhou and X. Zheng, "A general expression of magnetic force for soft ferromagnetic plates in complex magnetic fields,"
International Journal of Engineering Science, vol. 35, no. 15, pp. 1405-1417, 1997.

[10] Y. H. Zhou and K. Miya, "A theoretical prediction of natural frequency of a ferromagnetic beam plate with low susceptibility in an in-plane magnetic field," Journal of Applied Mechanics, vol. 65, no. 1, pp. 121-126, 1998.

[11] X. Wang, G. Lu, and S. R. Guillow, "Magnetothermodynamic stress and perturbation of magnetic field vector in a solid cylinder," Journal of Thermal Stresses, vol. 25, no. 10, pp. 909-926, 2002.

[12] X. Wang, J. S. Lee, and X. Zheng, "Magneto-thermo-elastic instability of ferromagnetic plates in thermal and magnetic fields," International Journal of Solids and Structures, vol. 40, no. 22, pp. 6125-6142, 2003.

[13] X. Wang and H. L. Dai, "Magnetothermodynamic stress and perturbation of magnetic field vector in an orthotropic thermoelastic cylinder," International Journal of Engineering Science, vol. 42, no. 5-6, pp. 539-556, 2004.

[14] L. Librescu, D. Hasanyan, Z. Qin, and D. R. Ambur, "Nonlinear magnetothermoelasticity of anisotropic plates immersed in a magnetic field," Journal of Thermal Stresses, vol. 26, no. 11-12, pp. 1277-1304, 2003.

[15] X. Wang, J. S. Lee, and X. Zheng, "Magneto-thermo-elastic instability of ferromagnetic plates in thermal and magnetic fields," International Journal of Solids and Structures, vol. 40, no. 22, pp. 6125-6142, 2003.

[16] X. Wang and H. L. Dai, "Magnetothermodynamic stress and perturbation of magnetic field vector in an orthotropic thermoelastic cylinder," International Journal of Engineering Science, vol. 42, no. 5-6, pp. 539-556, 2004.

[17] D. J. Hasanyan, L. Librescu, and D. R. Ambur, "A few results on the foundation of the theory and behavior of nonlinear magnetoelastic plates carrying an electrical current," International Journal of Engineering Science, vol. 42, no. 15-16, pp. 15471572, 2004.

[18] X. Wang and J. S. Lee, "Dynamic stability of ferromagnetic beam-plates with magnetoelastic interaction and magnetic damping in transverse magnetic fields," Journal of Engineering Mechanics, vol. 132, no. 4, pp. 422-428, 2006.

[19] O. Damisa, V. O. S. Olunloyo, C. A. Osheku, and A. A. Oyediran, "Static analysis of slip damping with clamped laminated beams," European Journal of Scientific Research, vol. 17, no. 4, pp. 455-475, 2007.

[20] O. Damisa, V. O. S. Olunloyo, C. A. Osheku, and A. A. Oyediran, "Dynamic analysis of slip damping in clamped layered beams with non-uniform pressure distribution at the interface," Journal of Sound and Vibration, vol. 309, no. 3-5, pp. 349-374, 2008.

[21] V. O. S. Olunloyo, C. A. Osheku, and O. Damisa, "Vibration damping in structures with layered viscoelastic beam-plate," Journal of Vibration and Acoustics, Transactions of the ASME, vol. 130, no. 6, Article ID 061002, 2008.

[22] V. O. S. Olunloyo, O. Damisa, C. A. Osheku, and A. A. Oyediran, "Analysis of the effects of laminate depth and material properties on the damping associated with layered structures in a pressurized environment," Transactions of the Canadian Society for Mechanical Engineering, vol. 34, no. 2, pp. 165-196, 2010.

[23] C. A. Osheku and O. Damisa, "Vibration of conducting twolayer sandwich homogeneous elastic beams in transverse magnetic fields part I," ASCE Journal of Aerospace Engineering ASENG-245. In press. 
[24] L. E. Goodman and J. H. Klumpp, "Analysis of slip damping with reference to turbine blade vibration," Journal of Applied Mechanics, vol. 23, pp. 421-429, 1956.

[25] O. Damisa, "Slip damping of Timoshenko beam close to resonance," Journal of Engineering Research, vol. 11, no. 1-2, pp. 13-26, 2003. 

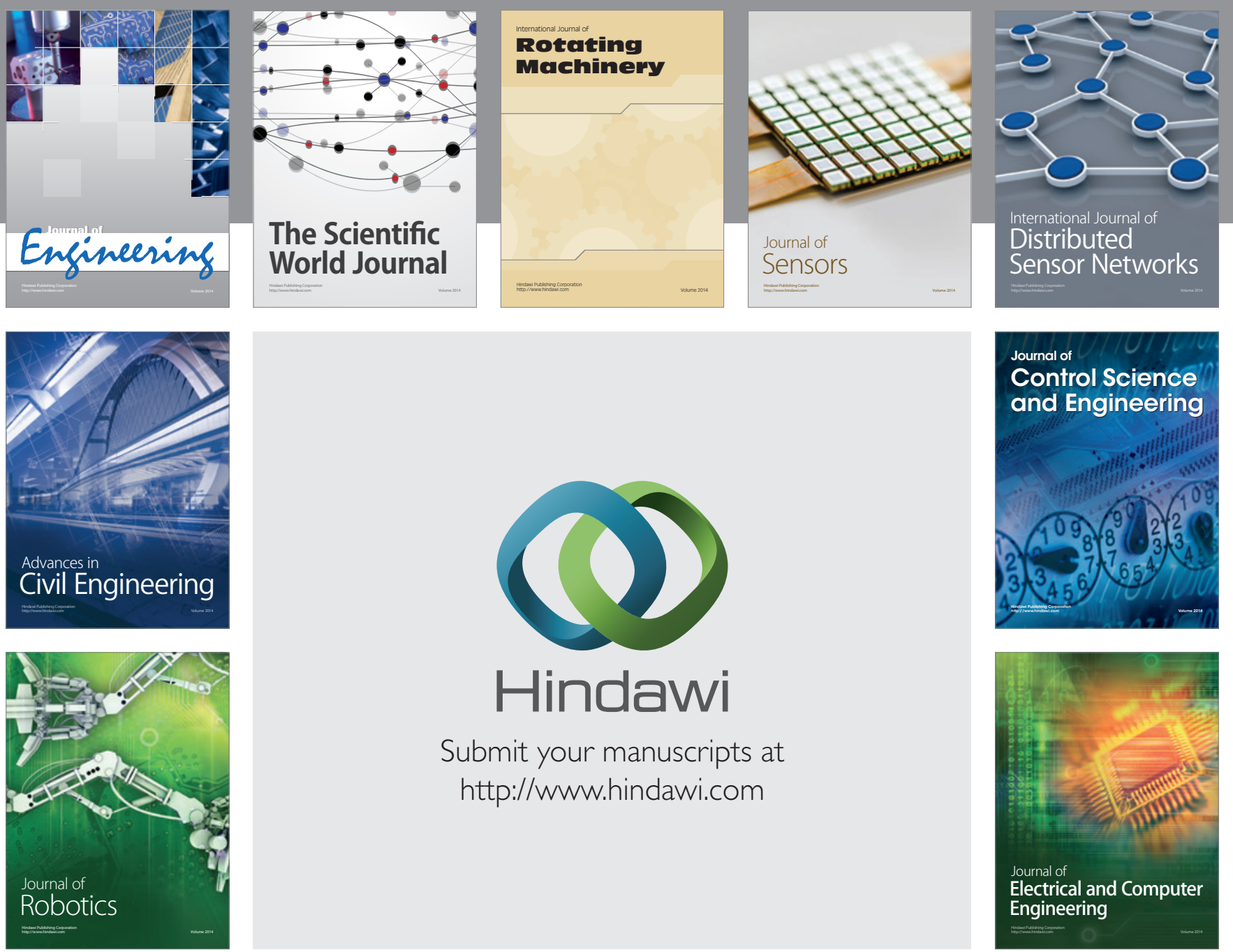

Submit your manuscripts at

http://www.hindawi.com
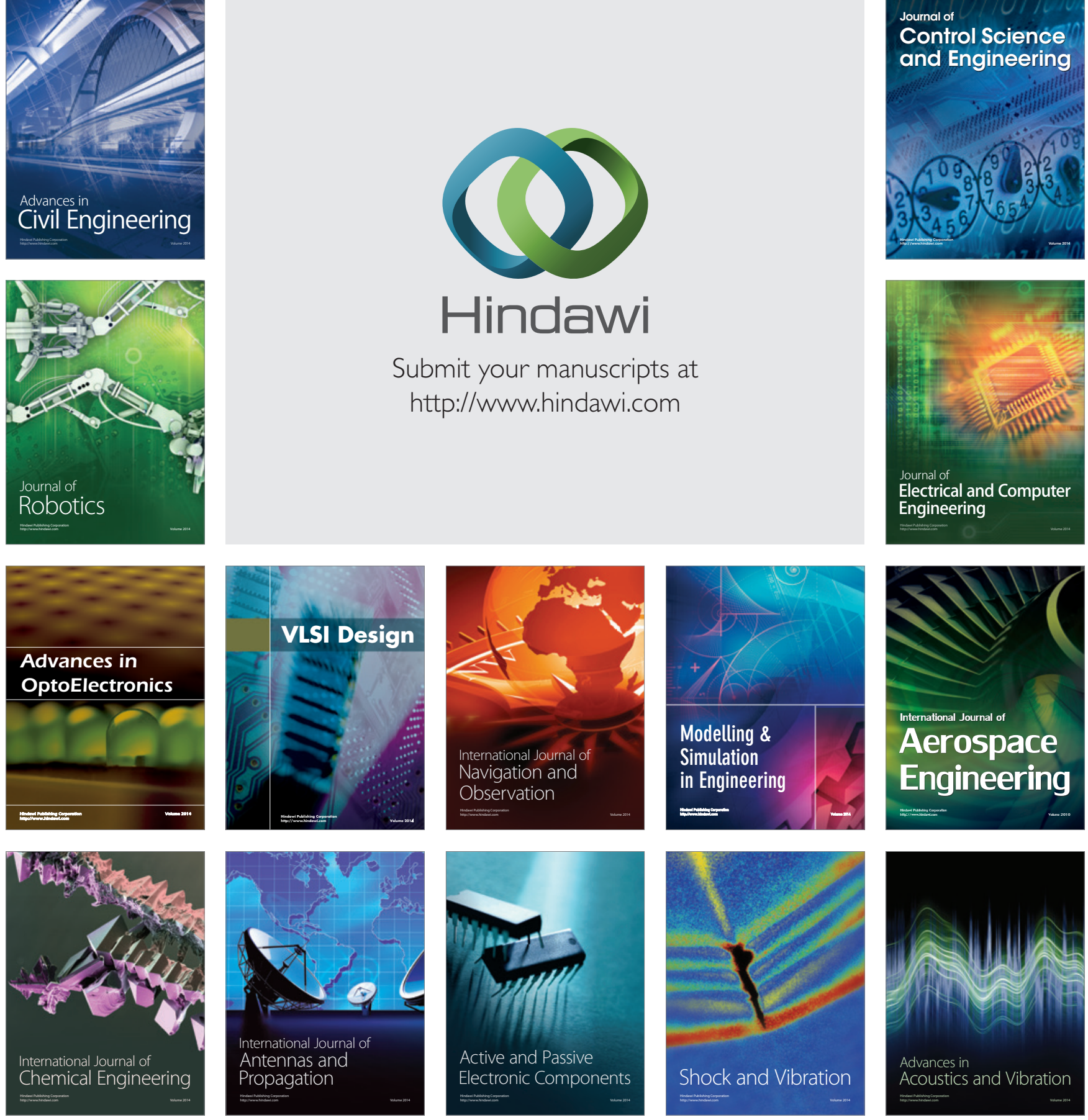